\title{
Weighted Residual Method for Diffraction of Plane P-Waves in a 2D Elastic Half-Space Revisited: On an Almost Circular Arbitrary-Shaped Canyon
}

\author{
Vincent W. Lee and Heather P. Brandow \\ Astani Civil and Environmental Engineering, University of Southern California, Los Angeles, CA 90089-2531, USA \\ Correspondence should be addressed to Vincent W. Lee; vlee@usc.edu
}

Received 2 May 2015; Accepted 12 July 2015

Academic Editor: Antonio Morales-Esteban

Copyright (C) 2015 V. W. Lee and H. P. Brandow. This is an open access article distributed under the Creative Commons Attribution License, which permits unrestricted use, distribution, and reproduction in any medium, provided the original work is properly cited.

\begin{abstract}
Scattering and diffraction of elastic in-plane P- and SV-waves by a surface topography such as an elastic canyon at the surface of a half-space is a classical problem which has been studied by earthquake engineers and strong-motion seismologists for over forty years. The case of out-of-plane SH-waves on the same elastic canyon that is semicircular in shape on the half-space surface is the first such problem that was solved by analytic closed-form solutions over forty years ago by Trifunac. The corresponding case of in-plane P- and SV-waves on the same circular canyon is a much more complicated problem because the in-plane P- and SVscattered-waves have different wave speeds and together they must have zero normal and shear stresses at the half-space surface. It is not until recently in 2014 that analytic solution for such problem is found by the author in the work of Lee and Liu. This paper uses the technique of Lee and Liu of defining these stress-free scattered waves to solve the problem of the scattering and diffraction of these in-plane waves on an on an almost-circular surface canyon that is arbitrary in shape.
\end{abstract}

\section{Introduction}

This paper studies the subject on the diffraction of in-plane P-waves in an elastic half-space by arbitrary-shaped canyons using the weighted residual method. It presents a solution for any arbitrary-shaped canyons where the depth of the canyon is approximately half the width of the canyon, such as a semicircle, ellipse, or trapezoid.

Researchers continue to study the effects of scattering and diffraction of waves on two-dimensional canyons in an elastic, isotropic, and homogeneous medium. These studies, which assist researchers to understand earthquake ground motions in and around topographic features, initially, addressed incident $\mathrm{SH}$-waves [1-3]. In solving $\mathrm{SH}$-waves, the method of images, which assumes equal and opposite scattered waves upon reflection, has been used [4]. Because of the mode conversion, an incident $\mathrm{P}$-wave, which produces both a reflected $\mathrm{P}$ - and SV-wave, is more complex. Thus, the P- and SV-waves diffraction problems cannot be solved using the method of images. Much of the latest and most recent work on diffraction and soil-structure interaction for topographies involving analytic methods is so far thus limited mostly to $\mathrm{SH}$-wave problems [5].

Recently in 2014, Lee and Liu analyzed the harmonic motion induced by an incident $\mathrm{P}$-wave for a two-dimensional diffraction around a semicircular canyon in an elastic halfspace using an analytic solution to satisfy the zero-stress boundary conditions [6]. In past approaches, numerical approximations of geometry and/or wave functions were made to satisfy the half-space boundary condition using wave functions that are a function of both sine and cosine [3, 7]. Lee and Liu's new method redefines the cylindrical-wave functions for both the longitudinal P- and shear SV-wave so that they are now a function of sine or cosine, but not a combination of both. Using the Fourier half-range expansion-and because each function itself is orthogonal in the half-space-the functions satisfy the zero-stress boundary condition along the half-space. The normal- and shear-stress boundary condition along the half-space in Figure 1 will be zero since the stress function contains $\sin (n \theta)$, which equals zero when $\theta=0, \pi$. 


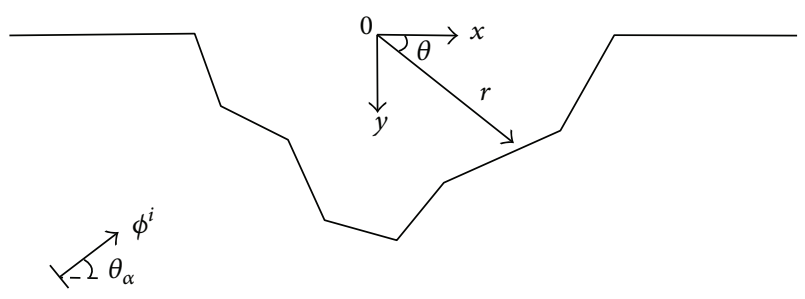

FIGURE 1: Arbitrary-shaped with coordinates at the half-space.

This paper expands on Lee and Liu's new approach for the semicircular canyon replacing it with an arbitrary-shaped canyon with harmonic motion induced by an incident Pwave. In this study, the weighted residual method is applied for the solution of the wave function for the arbitrary-shaped canyon $[8,9]$. Lee and Wu's method defined the scattered wave potentials as a combination of both the sine and the cosine functions, and the origin was defined above the halfspace for the case of the shallow canyon problem. This study uses the same principles from Lee and Liu's paper for which the P- and SV-cylindrical-wave functions are defined by the sine function only. In addition, Lee and $\mathrm{Wu}$ [9] define two sets of scattered P- and SV-waves, (a total of four sets of waves) so as to satisfy four sets of boundary conditions, two on the half-space and two on the canyon. The coordinate system in this study is located at the half-space surface, which allows for arbitrary-shaped canyons to be solved. The new method uses only one set of scattered P- and SV-waves, using the method of Lee and Liu [6] to automatically satisfy the freestress boundary conditions at the half-space surface. Using this improved weighted residual method, the results for Lee and Liu's semicircle were verified and new results for an ellipse, trapezoid, and rectangle are presented.

\section{Model}

The model for the canyon has no restrictions on shape other than the surface of the canyon which must be continuous and defined by a sequential number of points whose polar coordinates have an increasing value of $\theta$. In Figure 1 , the two-dimensional, arbitrary-shaped canyon in an elastic halfspace $(y>0)$ is defined. Each point will have an $(x, y)$ coordinate and once transformed into polar coordinates, each point will have an $(r, \theta)$ location. The geometry of the canyon is transformed from the rectangular coordinate system into a cylindrical coordinate system with the same origin at $O$, shown as follows:

$$
\begin{aligned}
& x=r \cos \theta, \\
& y=r \sin \theta, \\
& r=\sqrt{x^{2}+y^{2}}, \\
& \theta=\tan ^{-1}\left(\frac{y}{x}\right) .
\end{aligned}
$$

The figure encompasses the following: an incident plane Pwave defined by the potential $\phi^{i}$, with incidence angle $\theta_{\alpha}$.
The incident angle is measured with respect to the horizontal $x$-axis. The waves have a circular frequency $\omega=2 \pi f$, a longitudinal-wave velocity $\alpha$, and a transverse-wave velocity $\beta$.

The half-space is elastic, isotropic, and homogeneous, with the following material properties: Lame constants $\lambda$ and $\mu$ and mass density $\rho$. The longitudinal-wave velocity $\alpha$ and transverse-wave velocity $\beta$ are

$$
\begin{aligned}
& \alpha=\sqrt{\frac{\lambda+2 \mu}{\rho},} \\
& \beta=\sqrt{\frac{\mu}{\rho}} .
\end{aligned}
$$

Therefore, the constants $k_{\alpha}=\omega / \alpha$ (P-wave number) and $k_{\beta}=$ $\omega / \beta$ (SV-wave number) can be determined.

\section{Harmonic Motion Induced by Incident P-Wave}

Plane longitudinal- (P-) waves enter the half-space at angle $\theta_{\alpha}$, resulting in displacements and a propagation in the $x$ $y$ plane. The incident plane $\mathrm{P}$-wave is characterized by a potential $\phi^{i}$ and is defined in the $x-y$ plane as

$$
\phi^{i}=\exp \left(i k_{\alpha}\left(x \cos \theta_{\alpha}-y \sin \theta_{\alpha}\right)-i \omega t\right)
$$

The time factor is $\exp (-i \omega t)$, where $i=\sqrt{-1}$ and $t$ is the time coordinate. The time factor is removed from the equation because we are only interested in studying the amplitude of the waves as a function of the canyon geometry and not the time aspect $\omega t$, which describes the time-dependent oscillation of the wave due to the mode conversion along the half-space surface at $y=0$; the incident $\mathrm{P}$-wave produces a reflected plane $\mathrm{P}$-wave with $\mathrm{P}$-wave potential $\phi^{r}$, reflection angle $\theta_{\alpha}$, reflected plane SV-wave with a SV-wave potential $\psi^{r}$, and reflection angle $\theta_{\beta}$. The mode conversion takes place in order to satisfy the stress-free boundary conditions $\tau_{y y}=0$ and $\tau_{x y}=0$. The reflected $\mathrm{P}$ - and SV-wave potentials are derived in Achenbach's paper [10]. The reflection angles, $\theta_{\alpha}$ and $\theta_{\beta}$, are calculated using Snell's law, for $\alpha>\beta$ implies $\theta_{\alpha}>\theta_{\beta}$ :

$$
\frac{\sin \theta_{\alpha}}{\alpha}=\frac{\sin \theta_{\beta}}{\beta} .
$$

On the flat half-space surface, the stress-free boundary conditions can be satisfied for the incident $\mathrm{P}$ and the reflected Pand SV-wave by utilizing the boundary conditions:

$$
\begin{aligned}
& \sigma_{y}=\left.\tau_{y x}\right|_{y=0}=0, \\
& \sigma_{\theta}=\left.\tau_{\theta r}\right|_{\theta=0, \pi}=0 .
\end{aligned}
$$


This results in the following set of equations for the reflected $\mathrm{P}$-wave and SV-wave potentials defined in the $x-y$ plane as

$$
\begin{gathered}
\text { Reflected Plane P-Waves } \\
\phi^{r}=K_{1} \exp i k_{\alpha}\left(x \cos \theta_{\alpha}+y \sin \theta_{\alpha}\right), \\
\text { Reflected Plane SV-Waves } \\
\psi^{r}=K_{2} \exp i k_{\beta}\left(x \cos \theta_{\beta}+y \sin \theta_{\beta}\right) .
\end{gathered}
$$

The reflection coefficients $K_{1}$ and $K_{2}$ derived by Cao and Lee and are defined as [3]

$$
\begin{aligned}
& K_{1}=\left(\frac{\sin 2 \theta_{\alpha} \sin 2 \theta_{\beta}-\kappa^{2} \cos ^{2} 2 \theta_{\beta}}{\sin 2 \theta_{\alpha} \sin 2 \theta_{\beta}+\kappa^{2} \cos ^{2} 2 \theta_{\beta}}\right), \\
& K_{2}=\left(\frac{-\sin 2 \theta_{\alpha} \cos 2 \theta_{\beta}}{\sin 2 \theta_{\alpha} \sin 2 \theta_{\beta}+\kappa^{2} \cos ^{2} 2 \theta_{\beta}}\right) .
\end{aligned}
$$

The free-field waves are unaffected by the canyon and become a combination of the input $\mathrm{P}$-wave and reflected $\mathrm{P}$ - and SVwaves. The free-field P-wave potential $\phi^{\mathrm{ff}}$ and the free-field SV-wave potential $\psi^{\mathrm{ff}}$ are as follows:

$$
\begin{aligned}
\phi^{\mathrm{ff}}= & \phi^{i}+\phi^{r} \\
= & \exp i k_{\alpha}\left(x \cos \theta_{\alpha}-y \sin \theta_{\alpha}\right) \\
& +K_{1} \exp i k_{\alpha}\left(x \cos \theta_{\alpha}+y \sin \theta_{\alpha}\right), \\
\psi^{\mathrm{ff}}= & \psi^{r}=K_{2} \exp i k_{\beta}\left(x \cos \theta_{\beta}+y \sin \theta_{\beta}\right) .
\end{aligned}
$$

Both of these potentials must satisfy the zero-stress boundary conditions along the half-space surface; namely, for all $r \geq a$ (same as (5)),

$$
\begin{aligned}
& \sigma_{y}=\left.\tau_{y x}\right|_{y=0}=0, \\
& \sigma_{\theta}=\left.\tau_{\theta r}\right|_{\theta=0, \pi}=0 .
\end{aligned}
$$

The solution to the wave problems is to start by deriving the Helmholtz wave equation

$$
\nabla^{2} \phi+k_{\alpha}^{2} \phi=\frac{\partial^{2} \phi}{\partial r^{2}}+\frac{1}{r} \frac{\partial \phi}{\partial r}+\frac{1}{r^{2}} \frac{\partial^{2} \phi}{\partial \theta^{2}}+k_{\alpha}^{2} \phi=0
$$

Prior research has shown that the solution $\Theta(\theta)$ to the Helmholtz wave equation for the scattered P-wave potential $\phi=\phi(r, \theta) e^{-i \omega t}$ in 2D cylindrical (polar) coordinates in the half-space region $r \geq 0,0 \leq \theta \leq \pi$, and in the full space $-\pi \leq \theta \leq \pi$, is a combination of both the $\sin n \theta$ and $\cos n \theta$ terms included as two independent solutions with $A$ and $B$ as constants and shown as

$$
\Theta_{n}(\theta)=A_{n} \sin n \theta+B_{n} \cos n \theta .
$$

Lee and Liu [6] observed that in the half-space region, where $r \geq 0,0 \leq \theta \leq \pi$, the $\{\sin n \theta\}$ and $\{\cos n \theta\}$ terms are no longer two independent solutions. The authors concluded that the solution will either be a sine series or a cosine series, but not a combination of both. Therefore the solution becomes

$$
\Theta_{n}(\theta)=\sin n \theta \text { or } \cos n \theta \text {. }
$$

To solve the semicircular canyon, it is imperative that Sommerfeld's radiation condition (waves that are outgoing towards infinity) is satisfied. The Hankel function of the first kind, $H_{n}^{(1)}\left(k_{\alpha} r\right)$, is used since it represents an outgoing wave that decreases in amplitude, unlike the Hankel function of the second kind, $H_{n}^{(2)}\left(k_{\alpha} r\right)$, which represents an incoming wave.

Therefore, using the theory asserted by Lee and Liu [6] that, in the half-space, the sine functions are complete and orthogonal, the scattered wave potentials will take only the sine terms:

$$
\begin{aligned}
& \phi^{s}=\sum_{n=0}^{\infty} A_{n} H_{n}^{(1)}\left(k_{\alpha} r\right) \sin n \theta, \\
& \psi^{s}=\sum_{n=0}^{\infty} B_{n} H_{n}^{(1)}\left(k_{\beta} r\right) \sin n \theta
\end{aligned}
$$

with $A_{0}=B_{0}=0$. Here $H_{n}^{(1)}(\cdot)$ are Hankel functions of the first kind corresponding to outgoing waves to infinity satisfying Sommerfeld's radiation condition. They can be defined in terms of Bessel functions of the 1st and 2nd kind which in turn are computed by algorithms defined and found in Abramowitz and Stegun [11].

\section{Boundary Conditions for the Canyon Surface}

Boundary conditions on the canyon surface must be satisfied for the free-field and scattered wave potentials. To satisfy these boundary conditions for no force on the canyon surface, the traction components-radial $T_{r}$, and angular $T_{\theta}$-on the surface are computed (see Figure 2(a)). Both traction components must satisfy the condition that there is zero stress as derived in the papers by Lee and $\mathrm{Wu}[8,9]$. The equations for the traction components (14) are derived from the stress equation (15):

$$
\begin{aligned}
& T_{r}=\sigma_{r} \cos \alpha+\tau_{r \theta} \sin \alpha=0, \\
& T_{\theta}=\sigma_{r} \sin \alpha+\tau_{r \theta} \cos \alpha=0
\end{aligned}
$$

In Figure 2(b), the angle $\alpha$ is the angle between the radial vector $\widehat{r}$ and the normal vector $\widehat{n}$ at each point of the surface that is measured positive in the counterclockwise direction from the radial vector. The equations for the stresses are defined as $[10,12]$

$$
\begin{aligned}
& \sigma_{r}=\lambda \nabla^{2} \phi+2 \mu\left[\frac{\partial^{2} \phi}{\partial r^{2}}+\frac{\partial}{\partial r}\left(\frac{1}{r} \frac{\partial \psi}{\partial \theta}\right)\right], \\
& \sigma_{\theta}=\lambda \nabla^{2} \phi
\end{aligned}
$$




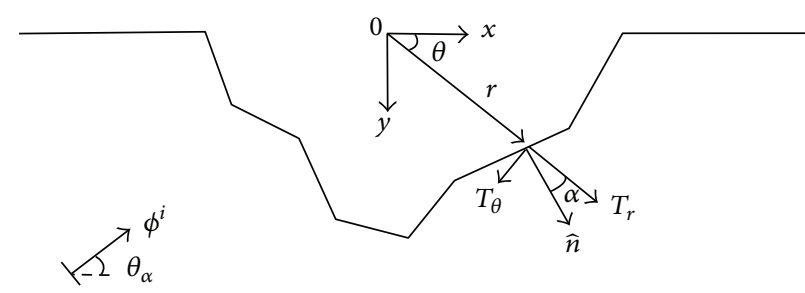

(a)

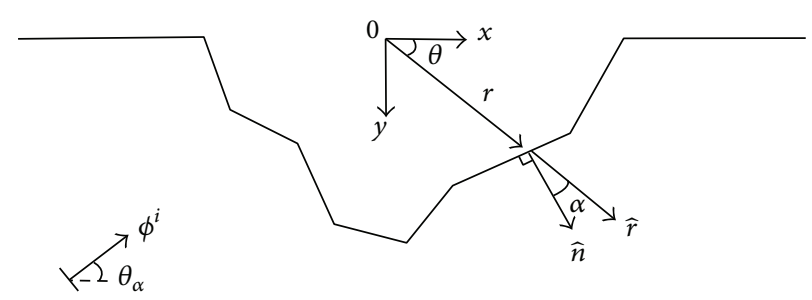

(b)

Figure 2: Traction components.

$$
\begin{array}{r}
+2 \mu\left[\frac{1}{r}\left(\frac{\partial \phi}{\partial r}+\frac{1}{r} \frac{\partial^{2} \phi}{\partial \theta^{2}}\right)+\frac{1}{r}\left(\frac{\partial \psi}{\partial \theta}-\frac{\partial^{2} \psi}{\partial r \partial \theta}\right)\right], \\
\tau_{r \theta}=\mu\left[\frac{2}{r} \frac{\partial^{2} \phi}{\partial r \partial \theta}-\frac{2}{r^{2}} \frac{\partial \phi}{\partial \theta}+\frac{1}{r^{2}} \frac{\partial^{2} \psi}{\partial \theta^{2}}-\frac{\partial^{2} \psi}{\partial r^{2}}+\frac{1}{r} \frac{\partial \psi}{\partial r}\right] .
\end{array}
$$

The stresses $\tau_{r \theta}^{i+r}, \sigma_{r}^{i+r}, \sigma_{\theta}^{i+r}$ are the in-plane stresses induced by the incident $\mathrm{P}$-wave and reflected $\mathrm{P}$-wave and $\mathrm{SV}$-wave potentials. These stresses are calculated directly from (15) and by substituting the free-field potentials in (8).

The stresses due to the scattered waves $\sigma_{r}^{s}, \tau_{r \theta}^{s}, \sigma_{\theta}^{s}$ are calculated by substituting the equations for the potentials of (13) into (15) and shown as

$$
\begin{aligned}
\sigma_{r}^{s} & =\frac{2 \mu}{r^{2}}\left(\sum_{n=1}^{\infty} A_{n} E_{11}^{(3)}\left(n, k_{\alpha} r\right) \sin n \theta\right. \\
& \left.+C_{n} E_{12}^{(3)}\left(n, k_{\beta} r\right) \cos n \theta\right), \\
\sigma_{\theta}^{s} & =\frac{2 \mu}{r^{2}}\left(\sum_{n=1}^{\infty} A_{n} E_{21}^{(3)}\left(n, k_{\alpha} r\right) \sin n \theta\right. \\
& \left.+C_{n} E_{22}^{(3)}\left(n, k_{\beta} r\right) \cos n \theta\right), \\
\tau_{\theta}^{s} & =\frac{2 \mu}{r^{2}}\left(\sum_{n=1}^{\infty} A_{n} E_{41}^{(3)}\left(n, k_{\alpha} r\right) \cos n \theta\right. \\
& \left.+C_{n} E_{42}^{(3)}\left(n, k_{\beta} r\right) \sin n \theta\right)
\end{aligned}
$$

with $E_{i j}^{(3)}$ the stress functions given by [12]. The tractions on the half-space surface due to the scattered waves, incident waves, and reflected waves are all given by

$$
\begin{gathered}
\left.\sigma_{y}\right|_{y=0}=\left.\sigma_{\theta}\right|_{\theta=0, \pi}=0, \\
\left.\tau_{y x}\right|_{y=0}=\left.\tau_{\theta r}\right|_{\theta=0, \pi}=0
\end{gathered}
$$

for both free-field and scattered waves.
On the canyon, the boundary condition of zero stress that must be satisfied on the surface of the arbitrary shape is [9] as follows:

$$
\begin{aligned}
& T_{r}^{i+r}+T_{r}^{s}=0, \\
& T_{\theta}^{i+r}+T_{\theta}^{s}=0 .
\end{aligned}
$$

\section{Application of Weighted Residual Method}

The functions that define the stresses are an infinite summation. Therefore, the traction equations are an infinite summation. An approximate solution would use a finite summation with $N$ terms and thus $2 N$ unknowns, $A_{n}$, and $C_{n}, n=1$ to $N$. The procedure for solving these two equations, traction equation (18), is a special case of the method of moments defined in Roger Harrington's paper, "Matrix methods for field problems" [13]. A set of weighting functions, $w_{1}, w_{2}, w_{3}, \ldots, w_{N}$, in the range of 0 to $\pi$, is defined and applied to the traction terms. This results in $2 \mathrm{~N}$ equations that require integration from 0 to $\pi$. Applying the method of weighted residuals, the weighting function is chosen as

$$
w_{m}=\sin m \theta \text {. }
$$

For $m=1,2, \ldots, N$, the function is

$$
\begin{aligned}
& \int_{\theta}\left(T_{r}^{S}\right)_{N} w_{m} d \theta=-\int_{\theta}\left(T_{r}^{i+r}\right)_{N} w_{m} d \theta \\
& \int_{\theta}\left(T_{\theta}^{S}\right)_{N} w_{m} d \theta=-\int_{\theta}\left(T_{\theta}^{i+r}\right)_{N} w_{m} d \theta
\end{aligned}
$$

Since $\sin (m \theta)$ equals zero when $m=0,(20)$ represent $2 N$ pairs of equations with $2 N$ unknowns, $A_{n}$ and $C_{n}$, for $n=$ $1,2, \ldots, N$.

\section{Numerical Solutions}

The equations for traction, (20), form a set of complex simultaneous equations with unknowns $A_{n}$ and $C_{n}$, and 
coefficient matrix with terms $a_{i j}$, $c_{i j}$ and constant terms $r_{i}$, are shown as

$$
\begin{gathered}
{\left[\begin{array}{cccccc}
a_{11} & \cdots & a_{1 N} & c_{11} & \cdots & c_{1 N} \\
\vdots & \ddots & & & & \vdots \\
\vdots & & \ddots & & & \vdots \\
\vdots & & & \ddots & & \vdots \\
\vdots & & & & \ddots & \vdots \\
a_{2 N, 1} & \cdots & a_{2 N, N} & c_{2 N, 1} & \cdots & c_{2 N, N}
\end{array}\right]\left(\begin{array}{c}
A_{1} \\
\vdots \\
r_{1} \\
\vdots \\
r_{N} \\
r_{N+1} \\
\vdots \\
r_{2 N}
\end{array}\right)} \\
=\left(\begin{array}{c}
A_{N} \\
C_{1} \\
\vdots \\
\end{array}\right)
\end{gathered}
$$

The terms of the coefficient matrix are determined by substituting the stress equations for the scattered waves (16) into the traction equations, $T_{r}$ and $T_{\theta}$, (17) and multiplying each equation by the weighting function (19). Each term requires integration along the boundary of the canyon from 0 to $\pi$. The equations are integrated using the Gaussian quadrature method. The surface of integration-the canyon surfaceis divided into 400 segments defined by 401 points. Each segment is subdivided again by the 10-point Gauss-Legendre integration. This integration technique is highly accurate when the integrand is very smooth, which is the case for the sine and cosine functions, as the method converges much more quickly than in other integration schemes [14].

The constant terms $r_{1}$ to $r_{2 N}$ are calculated from tractions computed from the free-field waves. These free-field stresses are substituted into the equations for traction (17) and then multiplied by the weighting function (19) and integrated along the boundary from 0 to $\pi$.

The set of $2 N$ complex equations are solved for the unknowns $A_{1}$ to $A_{N}$, and $C_{1}$ to $C_{N}$.

\section{Comparison of Results to Previous and Existing Studies}

From the above analysis, which determined the coefficient $A_{n}$ and $C_{n}$ of the P- and SV-wave potentials, the displacement amplitudes can now be determined. These displacement amplitudes of interest occur on the surface of the half-space and within the canyon surface. The amplitudes are important in studying the variability of ground motions in the vicinity of a canyon.
The free-field displacements are calculated by substituting the incident- and reflected-wave potentials into

$$
\begin{aligned}
& u_{x}^{\mathrm{ff}}=\frac{\partial \varphi^{\mathrm{ff}}}{\partial x}+\frac{\partial \psi^{\mathrm{ff}}}{\partial y}, \\
& u_{y}^{\mathrm{ff}}=\frac{\partial \varphi^{\mathrm{ff}}}{\partial y}-\frac{\partial \psi^{\mathrm{ff}}}{\partial x} .
\end{aligned}
$$

The scatter-wave displacements are calculated by substituting the $\mathrm{P}$ - and $\mathrm{SV}$-wave potentials into

$$
\begin{aligned}
& u_{r}^{S}=\frac{\partial \varphi^{S}}{\partial r}+\frac{1}{r} \frac{\partial \psi^{S}}{\partial \theta} \\
& u_{\theta}^{S}=\frac{1}{r} \frac{\partial \varphi^{S}}{\partial \theta}-\frac{\partial \psi^{S}}{\partial r} .
\end{aligned}
$$

The resulting displacements for the scatter waves are in the following form [12]:

$$
\begin{aligned}
u_{r}^{s}= & \frac{1}{r} \sum_{n=1}^{\infty} A_{n} E_{71}^{(3)}\left(n, k_{\alpha} r\right) \sin n \theta \\
& +C_{n} E_{72}^{(3)}\left(n, k_{\beta} r\right) \cos n \theta \\
u_{\theta}^{s}= & \frac{1}{r} \sum_{n=1}^{\infty} A_{n} E_{81}^{(3)}\left(n, k_{\alpha} r\right) \cos n \theta \\
& +C_{n} E_{82}^{(3)}\left(n, k_{\beta} r\right) \sin n \theta
\end{aligned}
$$

with the displacement functions [12] given by

$$
\begin{aligned}
E_{71}^{(3)} & =E_{71}^{(3)}\left(n, k_{\alpha} r\right)=k_{\alpha} r H_{n-1}^{(1)}\left(k_{\alpha} r\right)-n H_{n}^{(1)}\left(k_{\alpha} r\right), \\
E_{72}^{(3)} & =E_{72}^{(3)}\left(n, k_{\beta} r\right)=n H_{n}^{(1)}\left(k_{\beta} r\right), \\
E_{81}^{(3)} & =E_{81}^{(3)}\left(n, k_{\alpha} r\right)=n H_{n}^{(1)}\left(k_{\alpha} r\right), \\
E_{82}^{(3)} & =E_{82}^{(3)}\left(n, k_{\beta} r\right) \\
& =-\left[k_{\beta} r H_{n-1}^{(1)}\left(k_{\beta} r\right)-n H_{n}^{(1)}\left(k_{\beta} r\right)\right] .
\end{aligned}
$$

In order to transform the scatter-wave displacements $u_{r}, u_{\theta}$ in polar coordinates to displacements $u_{x}$ and $u_{y}$ in rectangular coordinates, a transformation is used. The transformation is as follows:

$$
\left\{\begin{array}{l}
u_{x} \\
u_{y}
\end{array}\right\}=\left[\begin{array}{cc}
\cos \theta & -\sin \theta \\
\sin \theta & \cos \theta
\end{array}\right]\left\{\begin{array}{l}
u_{r} \\
u_{\theta}
\end{array}\right\} .
$$

The total displacement amplitudes are a linear combination of the scattered and the free-field waves, and the result of the real $(\mathrm{Re})$ and imaginary $(\mathrm{Im})$ parts

$$
\begin{aligned}
& \left|u_{x}\right|=\left(\operatorname{Re}^{2}\left(u_{x}^{\mathrm{ff}}+u_{x}^{S}\right)+\operatorname{Im}^{2}\left(u_{x}^{\mathrm{ff}}+u_{x}^{S}\right)\right)^{1 / 2}, \\
& \left|u_{y}\right|=\left(\operatorname{Re}^{2}\left(u_{y}^{\mathrm{ff}}+u_{y}^{S}\right)+\operatorname{Im}^{2}\left(u_{y}^{\mathrm{ff}}+u_{y}^{S}\right)\right)^{1 / 2} .
\end{aligned}
$$




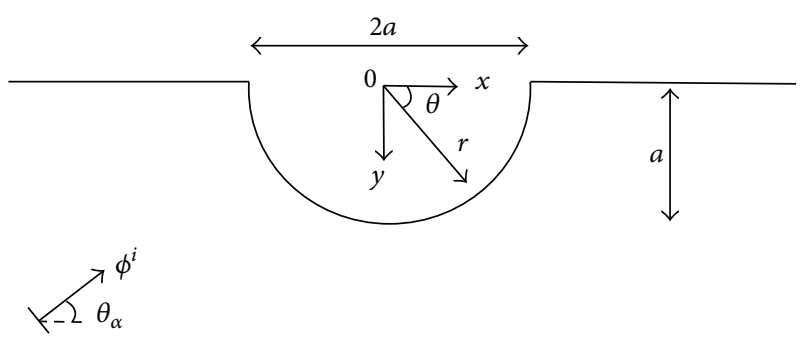

FIgURE 3: Semicircular canyon.

The phase angle of the points on the canyon and the surface of the half-space can be calculated by using

$$
\begin{aligned}
& \text { phase }_{x}=\tan ^{-1}\left[\frac{\operatorname{Im}\left(u_{x}\right)}{\operatorname{Re}\left(u_{x}\right)}\right], \\
& \text { phase }_{y}=\tan ^{-1}\left[\frac{\operatorname{Im}\left(u_{y}\right)}{\operatorname{Re}\left(u_{y}\right)}\right] .
\end{aligned}
$$

These two-dimensional displacement amplitudes, horizontal $\left(\left|u_{x}\right|\right)$ and vertical $\left(\left|u_{y}\right|\right)$, and the phase angle are plotted versus the dimensionless horizontal distance $x / a$ for a specific dimensionless frequency $\eta$ and an angle of incidence $\theta_{\alpha}$. The following plots use the dimensionless frequency parameters $\eta:$

$$
\eta=\frac{\omega a}{\pi \beta}=\frac{k_{\beta} a}{\pi}=\frac{2 a}{\lambda_{\beta}},
$$

where $a$ is the radius of the canyon, $\lambda_{\beta}$ is the wave length of the shear wave at frequency $\omega, \beta$ is the shear wave speed, $\kappa_{\beta}$ is the wave number, and $f$ is the cyclic frequency defined as $\omega=$ $2 \pi f$, with a Poisson ratio of $v=0.25$. To verify the validity of this numerical method, each plot was generated using an increasing value of $N_{\max }$-the total number of equationsuntil convergence was achieved. The figures have a depth-tohalf-width ratio of $h / a=1$ and their displacement amplitudes on the half-space surface and the surface of the canyon $\left(r_{1}=\right.$ a) are plotted along the horizontal $x$-axis in the interval $-4 \leq$ $x / a \leq 4$. The point $x / a=-1$ corresponds to the left rim of the canyon, $x / a=0$ to the bottom, and $x / a=1$ to the right rim. The incident $\mathrm{P}$-waves are assumed to arrive from the left $(x / a<0)$ in all cases. The displacement amplitudes were computed and compared with the results obtained by the closed-form analytic solutions presented in Lee and Liu [6] for the model of a semicircular canyon in Figure 3. The amplitudes from Lee and Liu [6] are shown in Figure 4, and the calculated results here are shown in Figure 5, to be described in the next paragraph.

In Figure 5, the results are shown for the circular canyon with a coordinate system at the half-space (Figure 3 ) for $\theta_{\alpha}=60, \eta=10$, and $N_{\max }=112$ using the weighted residual method verified by the results of Lee and Liu [6]. The results shown in Figure 5 can be compared with the results in Figure 4 for the incident $\mathrm{P}$-wave on a 2D semicircular canyon, which was computed using Lee and Liu's analytic solution with the matrix equations of order from $N=104-112$. The results matched Lee and Liu's results using $N=112$ terms.

\section{The Case of the Semicircular Canyon}

The results for the case of the semicircular canyon can also be compared theoretically by matching the boundary condition equations derived from the weighted residual method with those derived from Lee and Liu's exact closed-form solution [6]. For the incident $\mathrm{P}$-wave, the total waves are a combination of the free-field waves and scattered waves as follows:

$$
\begin{aligned}
\phi^{\mathrm{ff}} & =\sum_{n=0}^{\infty} J_{n}\left(k_{\alpha} a\right)\left(a_{n} \sin n \theta+b_{n} \cos n \theta\right), \\
\psi^{\mathrm{ff}} & =\sum_{n=0}^{\infty} J_{n}\left(k_{\beta} a\right)\left(c_{n} \sin n \theta+d_{n} \cos n \theta\right), \\
\phi^{s} & =\sum_{n=0}^{\infty} A_{n} H_{n}^{(1)}\left(k_{\alpha} r\right) \sin n \theta, \\
\psi^{s} & =\sum_{n=0}^{\infty} C_{n} H_{n}^{(1)}\left(k_{\beta} r\right) \sin n \theta .
\end{aligned}
$$

This assumes that the constants $a_{n}, b_{n}, c_{n}$, and $d_{n}$ are defined as follows:

$$
\begin{aligned}
& a_{n}=\sin n \theta_{\alpha}\left(K_{1}-1\right) \varepsilon_{n} i^{n}, \\
& b_{n}=\cos n \theta_{\alpha}\left(K_{1}+1\right) \varepsilon_{n} i^{n}, \\
& c_{n}=\sin n \theta_{\beta} K_{2} \varepsilon_{n} i^{n}, \\
& d_{n}=\cos n \theta_{\beta} K_{2} \varepsilon_{n} i^{n}
\end{aligned}
$$

and taking into consideration the stress-free boundary condition at the surface of the semicircular canyon at $r=a$,

$$
\begin{aligned}
& \int_{\theta}\left(\sigma_{r}^{s} \cos \alpha+\tau_{r \theta}^{s} \sin \alpha\right) \sin n \theta d \theta \\
& =-\int_{\theta}\left(\sigma_{r}^{\mathrm{ff}} \cos \alpha+\tau_{r \theta}^{\mathrm{ff}} \sin \alpha\right) \sin n \theta d \theta \\
& \int_{\theta}\left(\sigma_{\theta}^{s} \sin \alpha+\tau_{r \theta}^{s} \cos \alpha\right) \sin n \theta d \theta \\
& =-\int_{\theta}\left(\sigma_{\theta}^{\mathrm{ff}} \sin \alpha+\tau_{r \theta}^{\mathrm{ff}} \cos \alpha\right) \sin n \theta d \theta
\end{aligned}
$$

for the case of a semicircular canyon, $\alpha=0$. The orthogonality of the sine function gives

$$
\begin{aligned}
& \int_{0}^{\pi} \sin n \theta \sin m \theta= \begin{cases}\frac{\pi}{2} & m=n \\
0 & m \neq n,\end{cases} \\
& \int_{0}^{\pi} \cos n \theta \sin m \theta= \begin{cases}s_{m n}=\frac{2 m}{m^{2}-n^{2}} & m+n \text { odd } \\
0 & m+n \text { even. }\end{cases}
\end{aligned}
$$


Displacement amplitudes

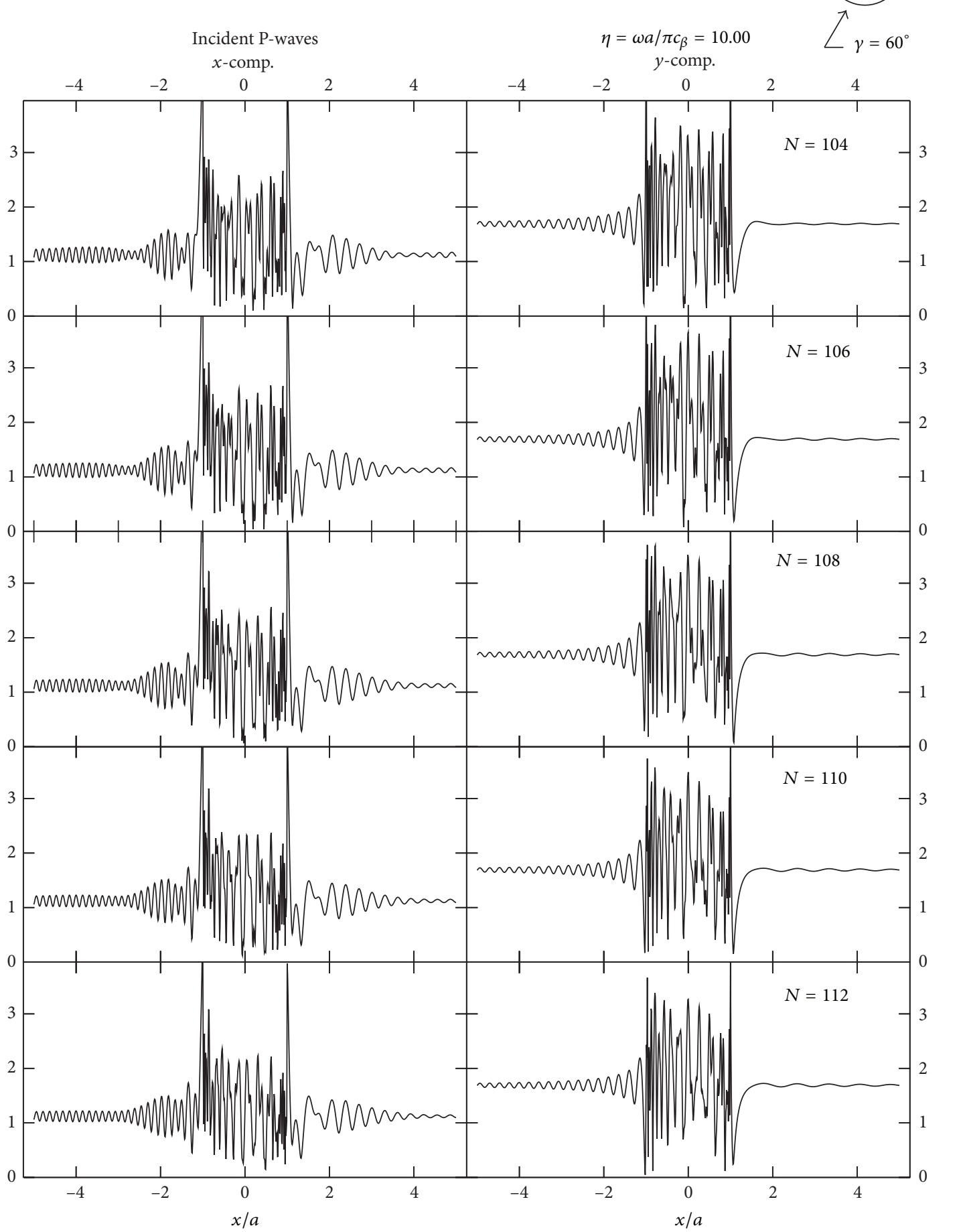

FIgURE 4: Arbitrary-shaped semicircular canyon $x / a$ versus $U x$ and $U y$ for $\eta=10, \theta=60^{\circ}$, and $N_{\max }=112$ (results from [6]).

Therefore, the two boundary condition equations reduce down to

$$
\begin{gathered}
\frac{\pi}{2} E_{11}^{(3)}(n) A_{n}+\sum_{\substack{m=1 \\
m+n \text { odd }}}^{\infty} E_{12}^{(3)}(m) s_{n m} C_{m}=s^{\mathrm{ff}}(n) \\
=-\frac{\pi}{2}\left(E_{11}^{(1)}(n) a_{n}-E_{12}^{(1)}(n) c_{n}\right)
\end{gathered}
$$

$$
\begin{gathered}
-\sum_{\substack{m=0 \\
m+n \text { odd }}}^{\infty}\left(E_{11}^{(1)}(m) b_{m}+E_{12}^{(1)}(m) d_{m}\right) s_{n m} \\
\sum_{\substack{m=1 \\
m+n \text { odd }}}^{\infty} E_{41}^{(3)}(m) s_{n m} A_{m}+\frac{\pi}{2} E_{42}^{(3)}(n) C_{n}=t^{\mathrm{ff}}(n)
\end{gathered}
$$



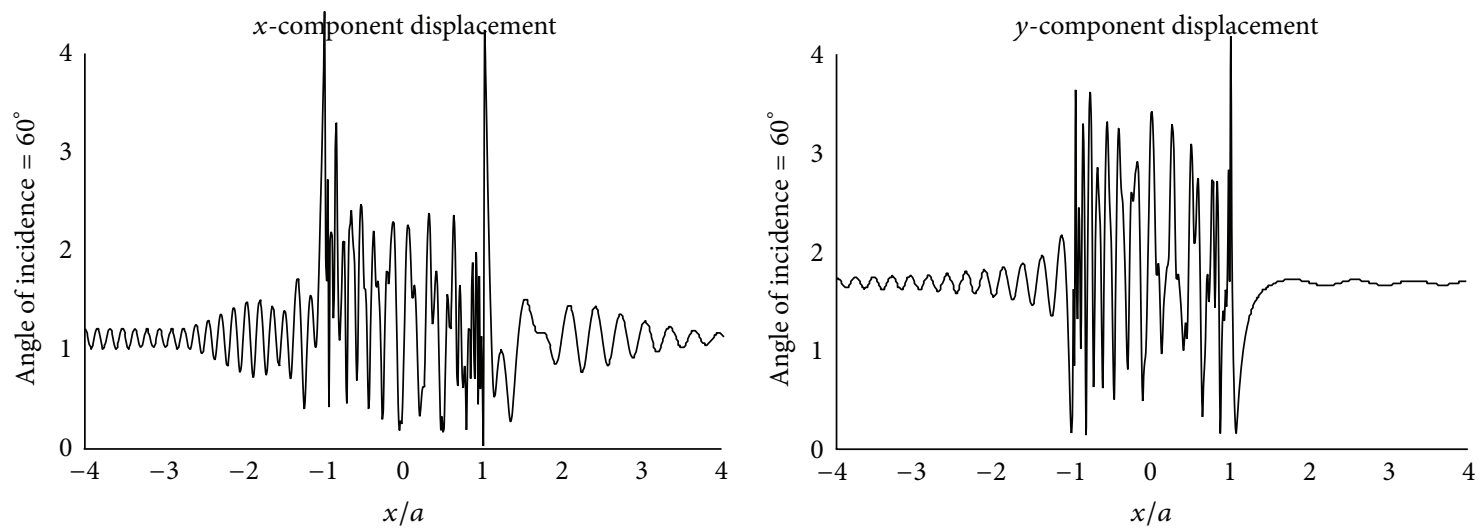

FIgURE 5: Weighted residual method recreated semicircular canyon matches Lee and Liu's results $\eta=10, \theta=60^{\circ}$, and $N_{\max }=126$.

$$
\begin{aligned}
= & -\frac{\pi}{2}\left(-E_{41}^{(1)}(n) b_{n}+E_{42}^{(1)}(n) d_{n}\right) \\
& -\sum_{\substack{m=1 \\
m+n \text { odd }}}^{\infty}\left(E_{41}^{(1)}(m) a_{m}+E_{42}^{(1)}(m) c_{m}\right) s_{n m} .
\end{aligned}
$$

Equations (34) are two boundary conditions that are identical to those derived in Lee and Liu's [6] exact solution for a semicircular canyon. Therefore, with the choice of the sine function as the weighting function, the weighted residual methods resulted in the exact closed-form solution for the case of a semicircular canyon in an elastic half-space.

\section{Application to Other Arbitrary Shapes}

This new methodology for solving the arbitrary-shaped canyon is applicable to any arbitrary-shaped canyon. This section of the paper will look at an elliptical-shaped canyon and a trapezoidal-shaped canyon. For each shape, the number of equations, $N_{\max }$, increases until convergence is reached or the problem becomes numerically unstable. The procedure that has been applied in this paper, the method of moments using weighting functions, is an approximate technique and its accuracy is a function of convergence as the infinite series are truncated to a finite number of unknown terms: $A_{1}, \ldots, A_{n}$ and $C_{1}, \ldots, C_{n}$. Three factors that affect the results are the number of terms, the stability of the Bessel functions, and integration. The cases of shallow canyons will be described and presented in the next paper.

9.1. Elliptical Canyon. The results for an elliptical canyon of depths $b / a=1.25$ and 1.5 , as shown in Figure 6 , are presented in this section. The incident $\mathrm{P}$-waves are assumed to arrive from the front side of the canyon for all cases. The results are presented in figures that show the $x$ - and $y$-displacement amplitudes for an angle of incidence of $\theta=5^{\circ}, 30^{\circ}, 60^{\circ}$, and $90^{\circ}$. The case for $\theta=30^{\circ}$ is for an oblique incident $\mathrm{P}$-wave and the case of $\theta=90^{\circ}$ is for a vertical incident $\mathrm{P}$-wave. The $x$ - and $y$-displacement amplitudes are plotted in the figures on the horizontal axis from $x / a=-4$ to $x / a=+4$. For convenience,

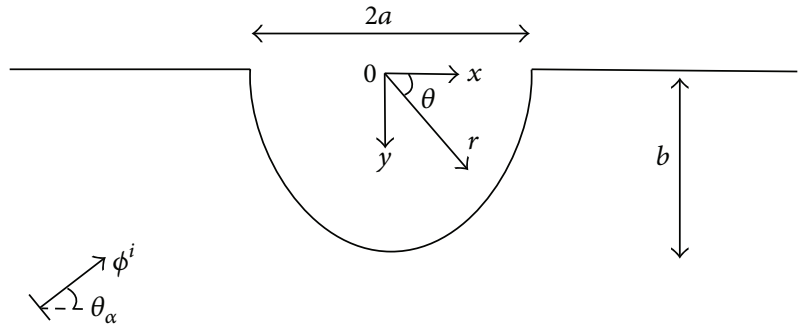

FIGURE 6: Arbitrary-shaped elliptical canyon.

each graph has three areas of interest: the front side (in which the waves arrive first $x / a=-4$ to $x / a=-1$ ), the backside (from $x / a=+1$ to $x / a=+4$ ), and the surface of the canyon (from $x / a=-1$ to $x / a=+1$ ). The results for $h=1.25$ are shown in Figures 7 and 8 and the results for $b=1.5$ are shown in Figures 9 and 10.

Figure 7 shows the results for an elliptical canyon of depth $b / a=1.25$, a dimensionless frequency of $\eta=2$, and an incident angle of $\theta=5^{\circ}, 30^{\circ}, 60^{\circ}$, and $90^{\circ}$. For the graphs with incident angle $\theta=5^{\circ}$, the $x$-component displacement amplitudes slightly oscillate about the free-field amplitude on the front side of the canyon and produce a shadowy behavior along the backside of the canyon. Within the canyon surface, both the $x$-and $y$-displacement amplitudes are oscillatory, producing a spike on the backside rim of the canyon at $x / a=+1$. The $y$-component displacement amplitudes slightly oscillate about the free-field amplitude on the front side of the canyon. On the backside of the canyon, the oscillations are very steady, showing a shadowy behavior. Within the canyon surface, the displacement amplitudes are oscillatory with a spike at the rims of the canyon at $x / a=$ +1 . For the graphs with incident angle $\theta=30^{\circ}$, the $x$ component displacement amplitudes tend to oscillate about the free-field amplitude on the front side of the canyon but gradually oscillate and produce a shadowy behavior along the backside of the canyon. Within the canyon surface both the $x$-and $y$-displacement amplitudes are highly oscillatory and produce a spike on the front side and backside rim of the canyons. The $y$-component displacement amplitudes 

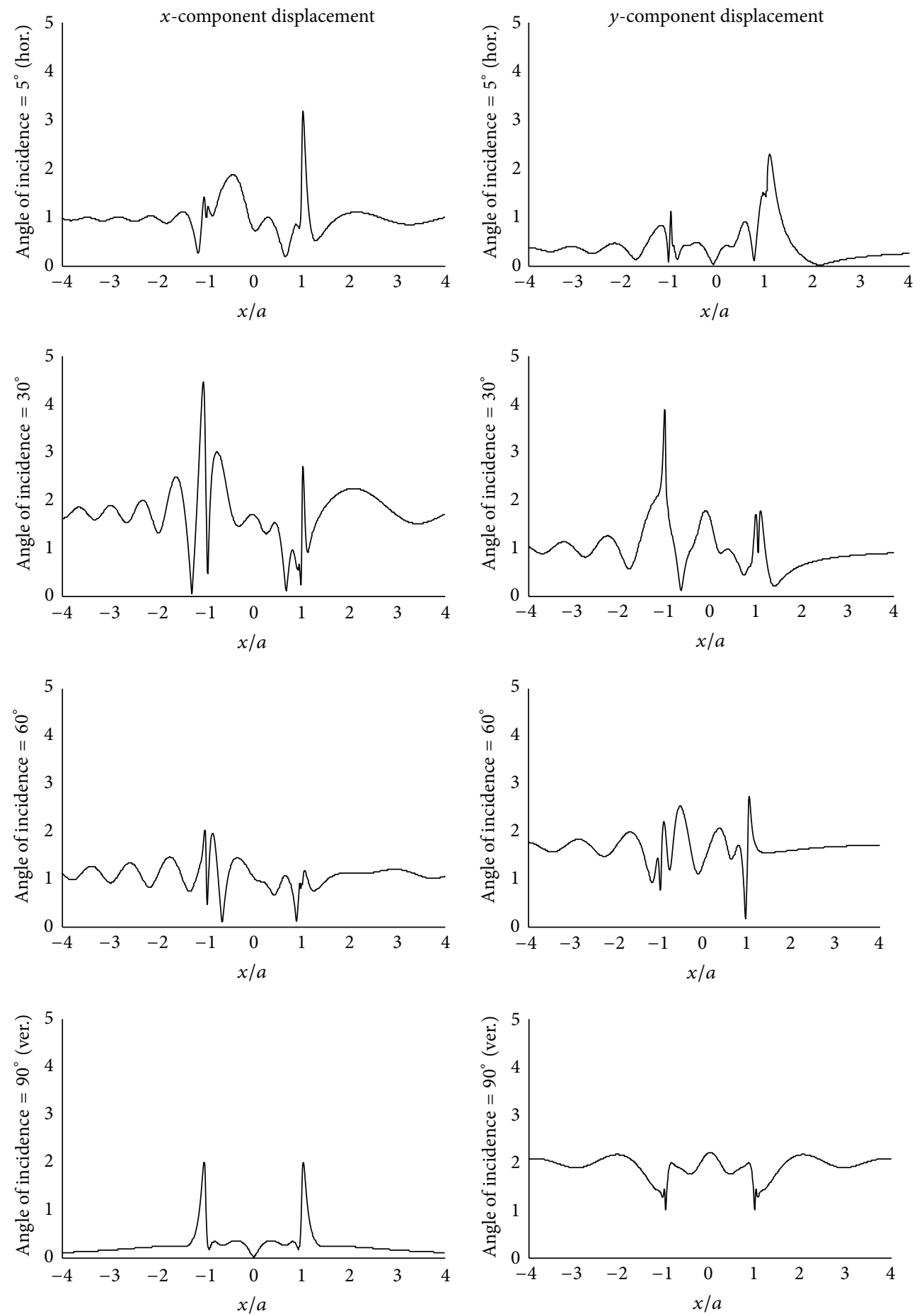

FIGURE 7: Ellipse-shaped canyon $b / a=1.25, \eta=2, \theta=5^{\circ}, 30^{\circ}, 60^{\circ}, 90^{\circ}$, and $N_{\max }=70$.

tend to oscillate about the free-field amplitude on the front side of the canyon and produce a shadowy behavior along the backside of the canyon. Within the canyon surface, the displacement amplitudes are highly oscillatory with spikes at the rims of the canyon $x / a=+1$ and $x / a=-1$. For the graphs with incident angle $\theta=60^{\circ}$, the $x$ - and $y$-component displacement amplitudes exhibit the same behavior as the graphs for incident angle $30^{\circ}$, with slightly smaller amplitudes and small corner spikes. For the graphs with incident angle of $\theta=90^{\circ}$, the $x$ - and $y$-component displacement amplitudes are symmetric about the origin 0 . The free-field amplitudes exhibit decaying oscillatory amplification on the front side of the canyon and shadowy behavior on the backside of the canyon. Within the canyon surface, the displacement 

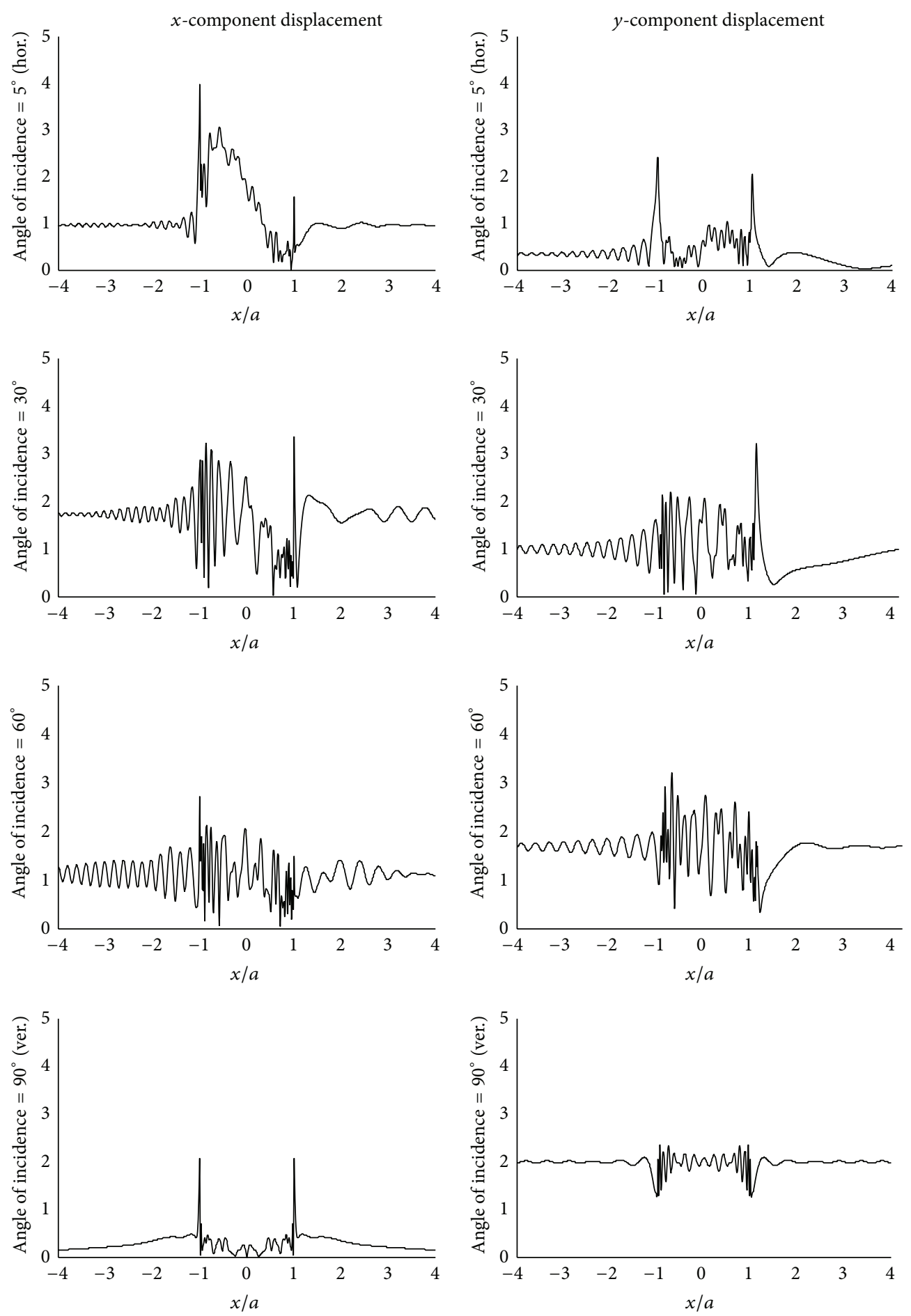

Figure 8: Ellipse-shaped canyon $b / a=1.25, \eta=8, \theta=5^{\circ}, 30^{\circ}, 60^{\circ}, 90^{\circ}$, and $N_{\max }=104$.

amplitudes are slightly oscillatory with spikes at both rims of the canyon for displacement of $x / a=+1$ and $x / a=-1$ for the $x$-component displacement amplitude.

Figure 8 shows the results for an elliptical canyon of depth $b / a=1.25$, dimensionless frequency $\eta=8$, and incidence angles of $\theta=5^{\circ}, 30^{\circ}, 60^{\circ}$, and $90^{\circ}$. For the graphs with incident angle $\theta=5^{\circ}$, the $x$-component displacement amplitudes oscillate about the free-field amplitude on the front side of the canyon and produce a shadowy behavior along the backside. Within the canyon surface, both the $x$ and $y$-displacement amplitudes are oscillatory, producing spikes on the front side of the canyon rim at $x / a=-1$ and the backside rim at $x / a=+1$. The $y$-component displacement amplitudes oscillate about the free-field amplitude on the 
$x$-component displacement
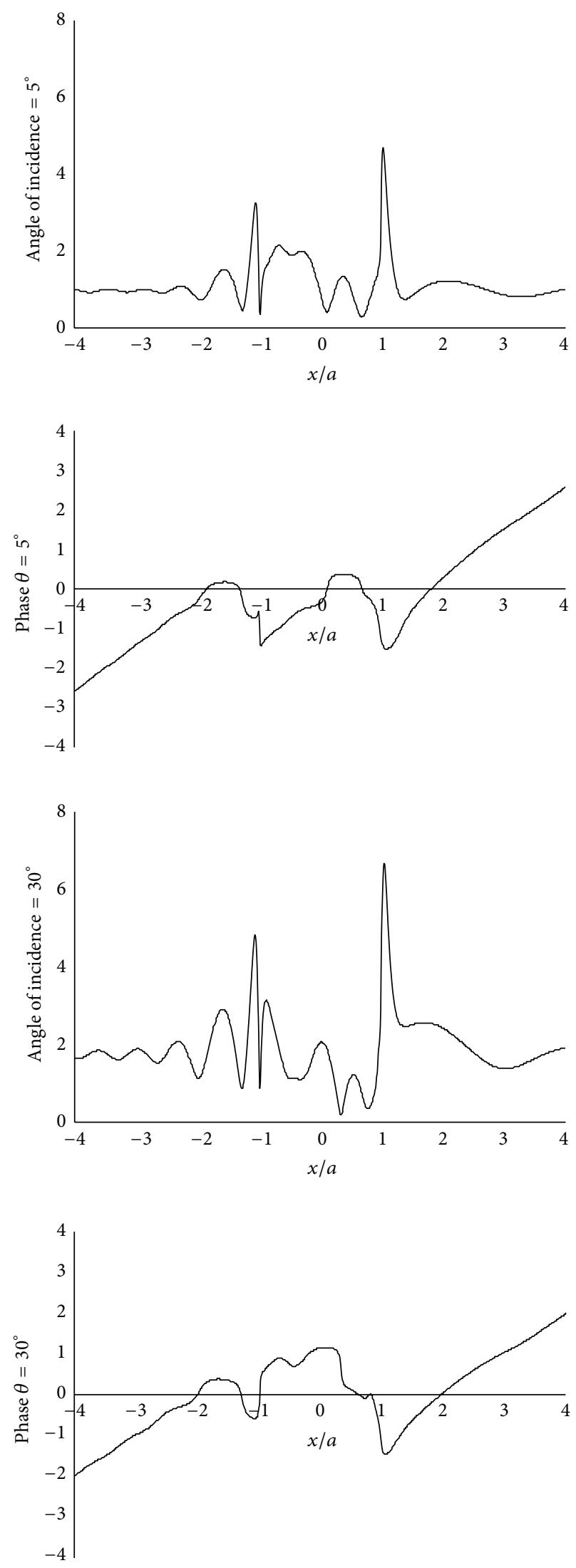

$y$-component displacement
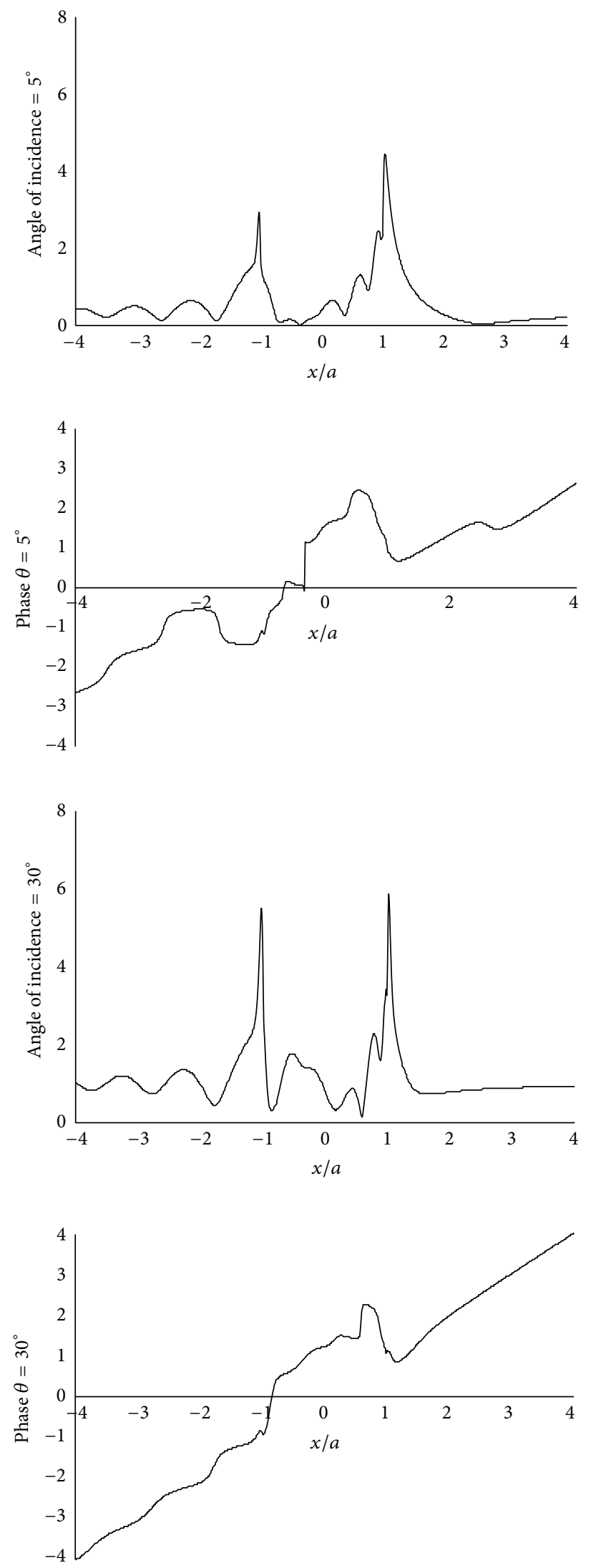

(a)

FIGURE 9: Continued. 

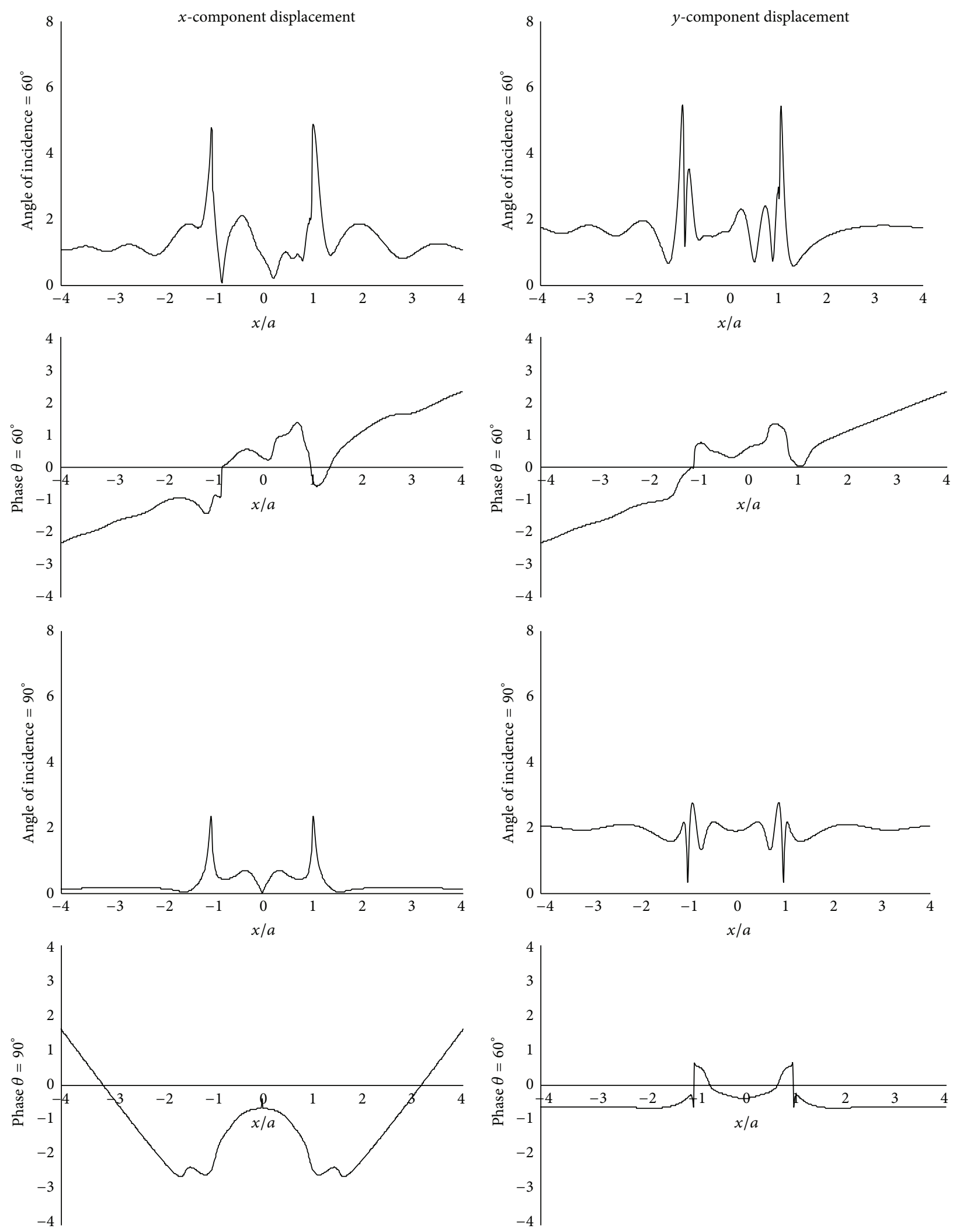

(b)

FIGURE 9: (a) Ellipse-shaped canyon $b / a=1.5, \eta=2, \theta=5^{\circ}, 30^{\circ}$, and $N_{\max }=60$. (b) Ellipse-shaped canyon $b / a=1.5, \eta=2, \theta=60^{\circ}, 90^{\circ}$, and $N_{\max }=60$. 

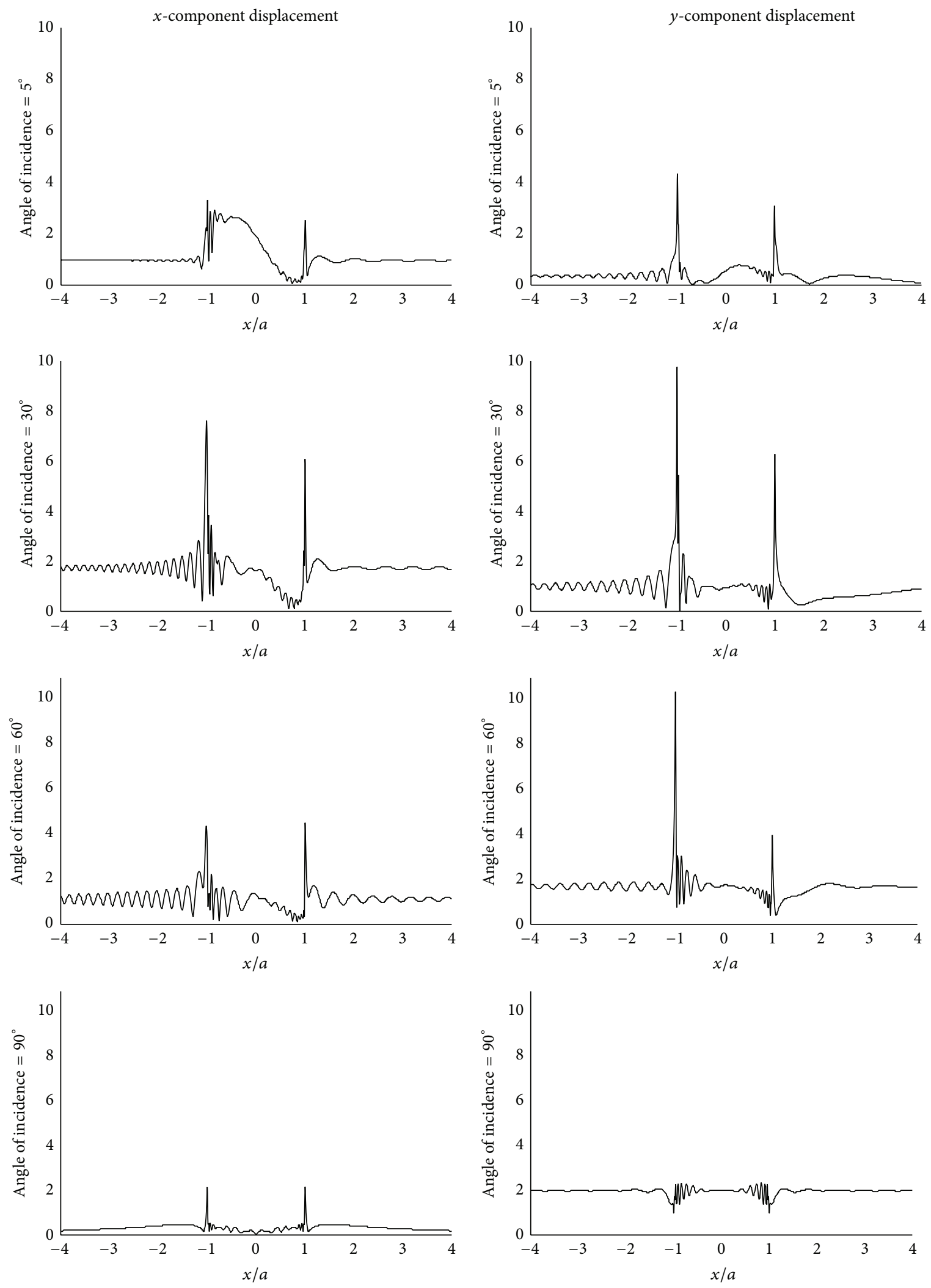

FIGURE 10: Ellipse-shaped canyon $b / a=1.5, \eta=8, \theta=5^{\circ}, 30^{\circ}, 60^{\circ}, 90^{\circ}$, and $N_{\max }=100$. 
front side and the backside of the canyon. The oscillations are very steady, showing a shadowy behavior. Within the canyon surface, the displacement amplitudes are oscillatory with spikes at the rims of the canyon at $x / a=+1$ and $x / a=-1$. For the graphs with incident angle $\theta=30^{\circ}$, the $x$-component displacement amplitudes tend to rapidly oscillate about the free-field amplitude on the front side of the canyon but gradually oscillate on the backside of the canyon. Within the canyon surface, both the $x$ - and $y$-displacement amplitudes are highly oscillatory and only produce a spike on the backside rim of the canyon at $x / a=+1$. The $y$ component displacement amplitudes tend to oscillate about the free-field amplitude on the front side of the canyon. On the backside of the canyon, the oscillations are very steady, showing a shadowy behavior. Within the canyon surface, the displacement amplitudes are highly oscillatory with spikes at the canyon rims at $x / a=+1$ and $x / a=-1$. These spikes are due to the sharp corners, which result in a change of curvature. For the graphs with incident angle $\theta=60^{\circ}$, the $x$ - and $y$-component displacement amplitudes exhibit the same behavior as the graphs for the incident angle of $30^{\circ}$ with slightly smaller amplitudes and no corner spike. For the graphs with incident angle $\theta=90^{\circ}$, the $x$ and $y$-component displacement amplitudes are symmetric about the origin 0 . The free-field amplitudes exhibit decaying oscillatory amplification on the front side of the canyon and a shadowy behavior on the backside of the canyon. Within the canyon surface, the displacement amplitudes are slightly oscillatory with spikes at both rims of the canyon for displacement of $x / a=+1$ and $x / a=-1$.

Figures 9(a) and 9(b) show the results for an elliptical canyon of depth $b / a=1.5$, a dimensionless frequency of $\eta=2$, and an incidence angle of $\theta=5^{\circ}, 30^{\circ}, 60^{\circ}$, and $90^{\circ}$. The displacement amplitudes tend to look similar along the free-field surface of the front side of the canyon and backside of the canyon as seen in Figure 7 for the ellipse with $b=1.25$. Figure 9 shows significant spikes at the rims of the canyons for an angle of incidence of $\theta=60^{\circ}$.

In Figure 9, the phase diagrams are shown alongside the corresponding displacement amplitudes. All phase diagrams have been scaled by $\pi$ and shifted arbitrarily to have a zero phase angle at $x / a=0$.

Figure 10 shows the results for elliptical canyon depth $b / a=1.5$, dimensionless frequency $\eta=8$, and angles of incidence $\theta=5^{\circ}, 30^{\circ}, 60^{\circ}$, and $90^{\circ}$. The displacement amplitudes tend to look similar along the free-field surface of the front side of the canyon and backside of the canyon as those seen in Figure 8 for the ellipse with $h=1.25$. Figure 8 shows significant spikes at the rims of the canyons and much less oscillatory behavior within the surface of the canyon, producing shadow-like behavior.

9.2. Trapezoidal Canyons. The results for the trapezoidal canyon, as shown in Figure 11, are presented in this section. The trapezoidal canyon is defined by $h / a=1$, with sloping sides of $60^{\circ}$ and $45^{\circ}$ measured from the horizontal axis. The incident $\mathrm{P}$-waves are assumed to arrive from the front side of the canyon for all cases. Each figure shows the $x$ - and $y$-displacement amplitudes for angles of incidence $\theta=30^{\circ}$,

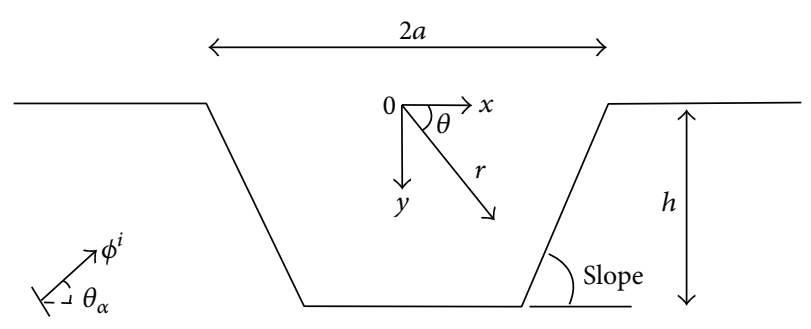

FIgURE 11: Trapezoidal canyon.

$60^{\circ}$, and $90^{\circ}$. The case for $\theta=30^{\circ}$ is for an oblique incident P-wave, and the case of $\theta=90^{\circ}$ is for a vertical incident $\mathrm{P}$-wave. The $x$ - and $y$-displacement amplitudes are plotted in the figures on the horizontal axis from $x / a=-4$ to $x / a=+4$. For convenience, each graph has three areas of focus: the front side, from $x / a=-4$ to $x / a-1$, the backside, from $x / a+1$ to $x / a=+4$, and the surface of the canyon, from $x / a=-1$ to $x / a+1$.

Figure 12 shows the results for a trapezoidal canyon with depth of $h / a=1$, dimensionless frequency $\eta=2$, a slope of $60^{\circ}$, and incidence angles of $\theta=30^{\circ}, 60^{\circ}$, and $90^{\circ}$. The $x$ - and $y$-displacement amplitudes are plotted in the figures on the horizontal axis from $x / a=-4$ to $x / a=+4$. For the graphs with incident angle $\theta=5^{\circ}$, the $x$-component displacement amplitudes gradually oscillate about the free-field amplitude on the front side of the canyon and produce a shadowy behavior along the backside of the canyon. Within the canyon surface, both the $x$ - and $y$-displacement amplitudes are oscillatory, producing clustered spikes at the canyon rims. The $y$ component displacement amplitudes oscillate about the freefield amplitude on the front side of the canyon. On the backside of the canyon, oscillations are very steady and show shadowy behavior. Within the canyon surface, the displacement amplitudes are oscillatory with clustered spikes at the canyon rims. For the graphs with incident angles $\theta=30^{\circ}$ and $\theta=60^{\circ}$, similar trends are observed for the $x$-component displacement amplitudes and $y$-component displacement amplitude with larger amplitude-clustered spikes on the front-sloping side of the canyon $(x / a=-1$ to $x / a=-0.5)$ and on the backsloping side of the canyon $(x / a=+0.5$ to $x / a=+1)$. For the graphs with incident angle $\theta=90^{\circ}$, the $x$-component displacement amplitudes are symmetric about the origin. They exhibit a shadowy behavior about the free-field amplitudes on the front side and backside of the canyon. Within the canyon surface, the displacement amplitudes are highly oscillatory with very large clustered spikes on the sloping sides. The $y$-component displacement amplitudes are symmetric about the origin and exhibit a shadowy behavior along the freefield amplitudes on the front side and backside of the canyon. Within the canyon surface, displacement amplitudes are highly oscillatory with clustered spikes on the sloping sides.

Figure 13 shows the results for a trapezoidal canyon of depth $h / a=1$, dimensionless frequency $\eta=6$, a slope of $60^{\circ}$, and incidence angles $\theta=30^{\circ}, 60^{\circ}$, and $90^{\circ}$. The $x$ - and $y$-displacement amplitudes are plotted in the figures on the horizontal axis from $x / a=-4$ to $x / a=+4$. 

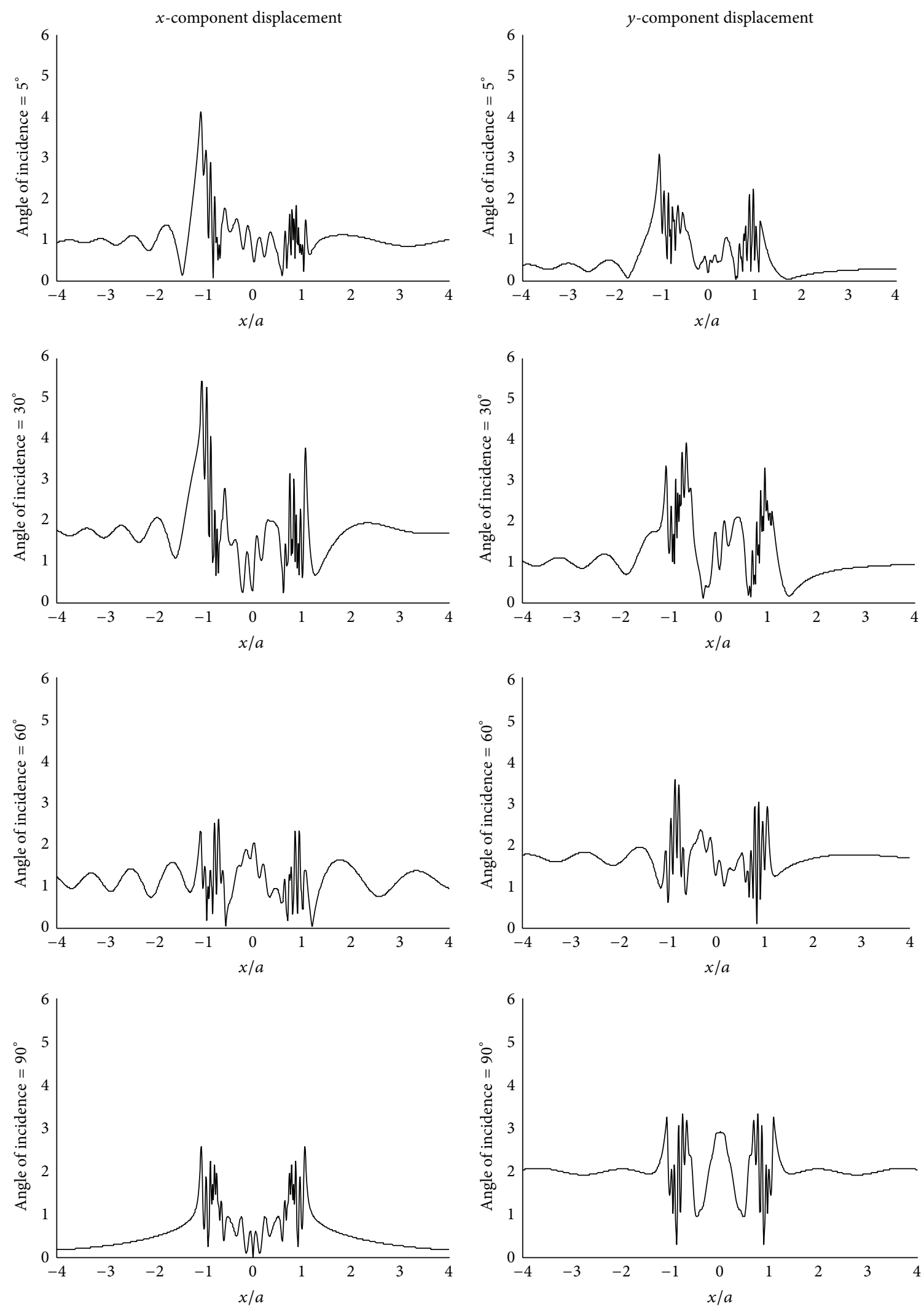

FIGURE 12: Trapezoid-shaped canyon $h / a=1, \eta=2, \theta=5^{\circ}, 30^{\circ}, 60^{\circ}, 90^{\circ}, N_{\max }=28$, and slope $60^{\circ}$. 

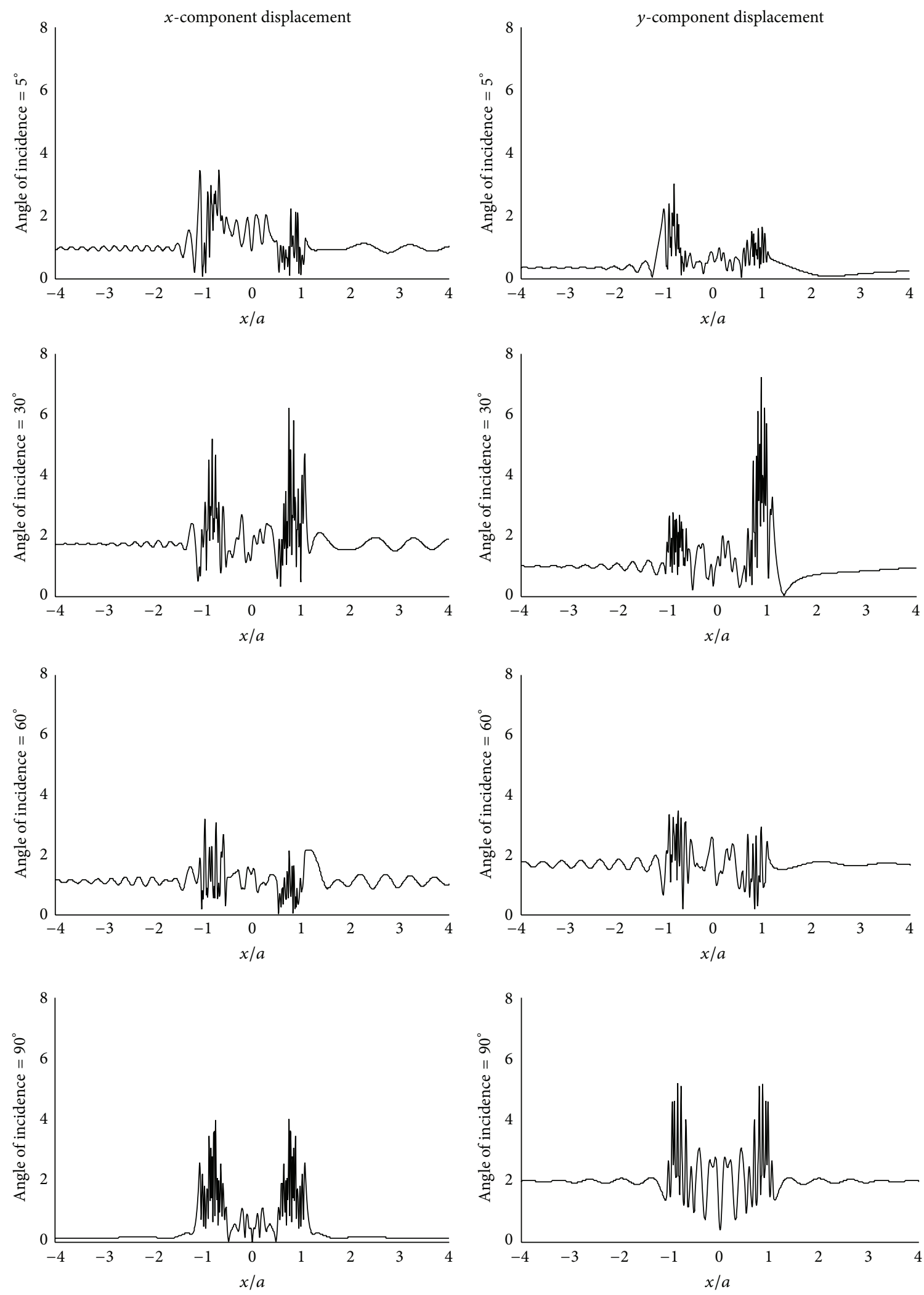

FIgURE 13: Trapezoid-shaped canyon $h / a=1, \eta=6, \theta=5^{\circ}, 30^{\circ}, 60^{\circ}, 90^{\circ}, N_{\max }=38$, and slope $60^{\circ}$. 
For the graphs with an incident angle of $\theta=5^{\circ}$, the $x$ component displacement amplitudes oscillate about the freefield amplitude on the front side of the canyon, and produce a shadowy behavior along the backside of the canyon. Within the canyon surface, both the $x$ - and $y$-displacement amplitudes are oscillatory, producing clustered spikes at the canyon rims. The $y$-component displacement amplitudes oscillate about the free-field amplitude on the front side of the canyon. On the backside of the canyon, the oscillations are very steady, showing a shadowy behavior. Within the canyon surface, the displacement amplitudes are oscillatory with clustered spikes at the rims of the canyon. For the graphs with incident angle $\theta=30^{\circ}$, the $x$-component displacement amplitudes tend to rapidly oscillate about the free-field amplitude on the front side of the canyon but gradually oscillate on the backside of the canyon. Within the canyon surface, the displacement amplitudes are highly oscillatory with clustered spikes on the front-sloping side $(x / a=-1$ to $x / a=-0.5)$ and on the back-sloping side of the canyon $(x / a=+0.5$ to $x / a=$ $+1)$. The oscillations are much more oscillatory than the ones observed for deep elliptical canyons. The $y$-component displacement amplitudes tend to oscillate about the free-field amplitude on the front side of the canyon. On the backside, the oscillations are very steady, showing a shadowy behavior. Within the canyon surface, the displacement amplitudes are highly oscillatory with small clustered spikes at the frontsloping side of the canyon and larger clustered spikes at the back-sloping side. The oscillations are at a higher frequency than the ones observed for the deep elliptical canyons. The graphs with incident angle $\theta=60^{\circ}$ display the same trends as the ones for incidence angle $\theta=30^{\circ}$ except without the spikes at the sloping sides of the trapezoidal canyon. For the graphs with incident angle $\theta=90^{\circ}$, the $x$-component displacement amplitudes are symmetric about the origin and exhibit no amplitude change about the free-field amplitudes on the front side and backside of the canyon. Within the canyon surface, the displacement amplitudes are highly oscillatory with very large clustered spikes on the sloping sides. The $y$-component displacement amplitudes are symmetric about the origin and exhibit a shadowy behavior along the free-field amplitudes on the front side and backside of the canyon. Within the canyon surface, displacement amplitudes are highly oscillatory with clustered spikes on the sloping sides.

Figure 14 shows the results for a trapezoidal canyon of depth $h / a=1$, dimensionless frequency $\eta=2$, a slope of $45^{\circ}$, and angles of incidence $\theta=5^{\circ}, 30^{\circ}, 60^{\circ}$, and $90^{\circ}$. For the graphs with incident angle $\theta=5^{\circ}$, the $x$-component displacement amplitudes gradually oscillate about the freefield amplitude on the front side of the canyon and produce a shadowy behavior along the backside of the canyon. Within the canyon surface both the $x$ - and $y$-displacement amplitudes are oscillatory. The $y$-component displacement amplitudes exhibit a very steady shadowy behavior on the front side and backside of the canyon. Within the canyon surface, the displacement amplitudes are oscillatory. For the graphs with the incident angles of $\theta=30^{\circ}$ and $\theta=60^{\circ}$, the $x$ - and $y$ component displacement amplitudes exhibit the same trends as those seen for the incident angle $\theta=5^{\circ}$. For the graphs with incident angle $\theta=90^{\circ}$, the $x$-component displacement amplitudes are symmetric about the origin and tend to exhibit a shadowy behavior along the free-field amplitudes on the front side and backside of the canyon. Within the canyon surface, the displacement amplitudes are highly oscillatory with very large clustered spikes at the rims of the canyon at $x / a=+1$ and $x / a=-1$. The $y$-component displacement amplitudes are symmetric about the origin and they also exhibit a shadowy behavior about the free-field amplitudes on the front side and backside of the canyon. Within the canyon surface, the displacement amplitudes are highly oscillatory with clustered spikes at the center of the canyon.

Figure 15 shows the results for a trapezoidal canyon of depth $h / a=1$, dimensionless frequency $\eta=6$, slope of $45^{\circ}$, and angles of incidence $\theta=5^{\circ}, 30^{\circ}, 60^{\circ}$, and $90^{\circ}$. For the graphs with an incident angle of $\theta=5^{\circ}$, the $x$ component displacement amplitudes oscillate about the freefield amplitude on the front side of the canyon and produce a shadowy behavior along the backside of the canyon. Within the canyon surface, both the $x$ - and $y$-displacement amplitudes are oscillatory, producing clustered spikes on the rims of the canyon rim. The $y$-component displacement amplitudes oscillate about the free-field amplitude on the front side of the canyon. On the backside of the canyon, the oscillations are very steady, showing a shadowy behavior. Within the canyon surface, the displacement amplitudes are oscillatory with clustered spikes that peak at the center of the canyon. For the graphs with incident angle $\theta=30^{\circ}$, the $x$ component displacement amplitudes slightly oscillate about the free-field amplitudes on the front side of the canyon and exhibit a shadowy behavior along the backside of the canyon. Within the canyon surface, the displacement amplitudes are highly oscillatory, oscillating much more rapidly than the ones observed for the trapezoidal canyons with a slope of $60^{\circ}$. The $y$-component displacement amplitudes exhibit a shadowy behavior about the free-field amplitudes on the front side and along the backside of the canyon. Within the canyon surface, the displacement amplitudes are highly oscillatory. For the graphs with incident angle $\theta=60^{\circ}$, the $x$ component displacement amplitudes rapidly oscillate about the free-field amplitudes on the front side of the canyon, but gradually oscillate on the backside of the canyon. Within the canyon surface, the displacement amplitudes are highly oscillatory with large clustered spikes at the rims of the canyon at $x / a=-1$ and $x / a=+1$. The $y$-component displacement amplitudes gradually oscillate about the freefield amplitudes on the front side of the canyon and along the backside of the canyon. Within the canyon surface, the displacement amplitudes are highly oscillatory. For the graphs with the incident angle $\theta=90^{\circ}$, the $x$-component displacement amplitudes are symmetric about the origin, and they tend to exhibit a shadowy behavior along the free-field amplitudes on the front side and the backside of the canyon. Within the canyon surface, the displacement amplitudes are highly oscillatory with very large clustered spikes at the canyon rims of $x / a=+1$ and $x / a=-1$. The $y$-component displacement amplitudes are symmetric about the origin, and they also exhibit a shadowy behavior about the freefield amplitudes on the front side and around the backside of the canyon. Within the canyon surface, the displacement 

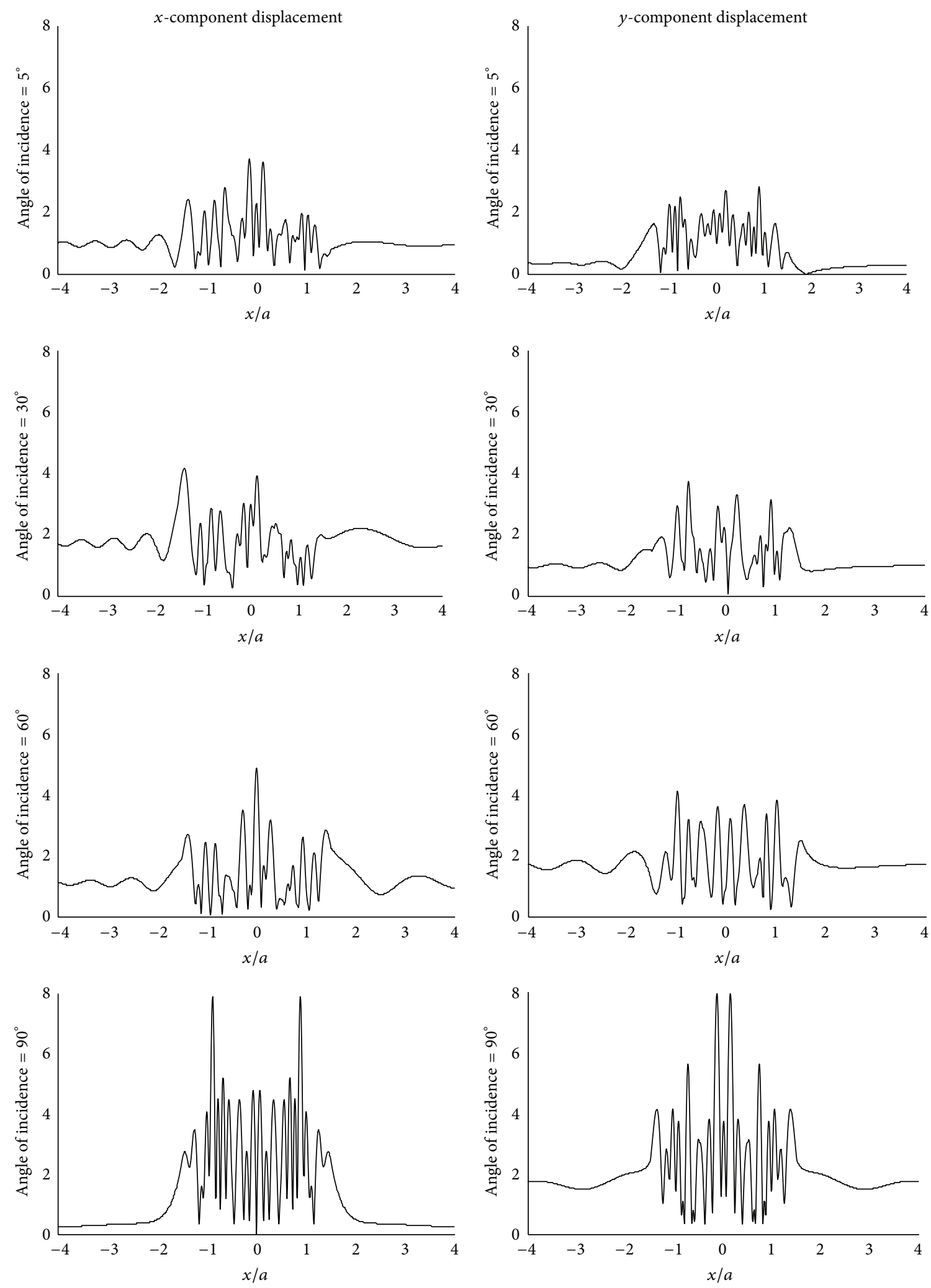

Figure 14: Trapezoid-shaped canyon $h / a=1, \eta=2, \theta=5^{\circ}, 30^{\circ}, 60^{\circ}, 90^{\circ}, N_{\max }=18$, and slope $45^{\circ}$. 

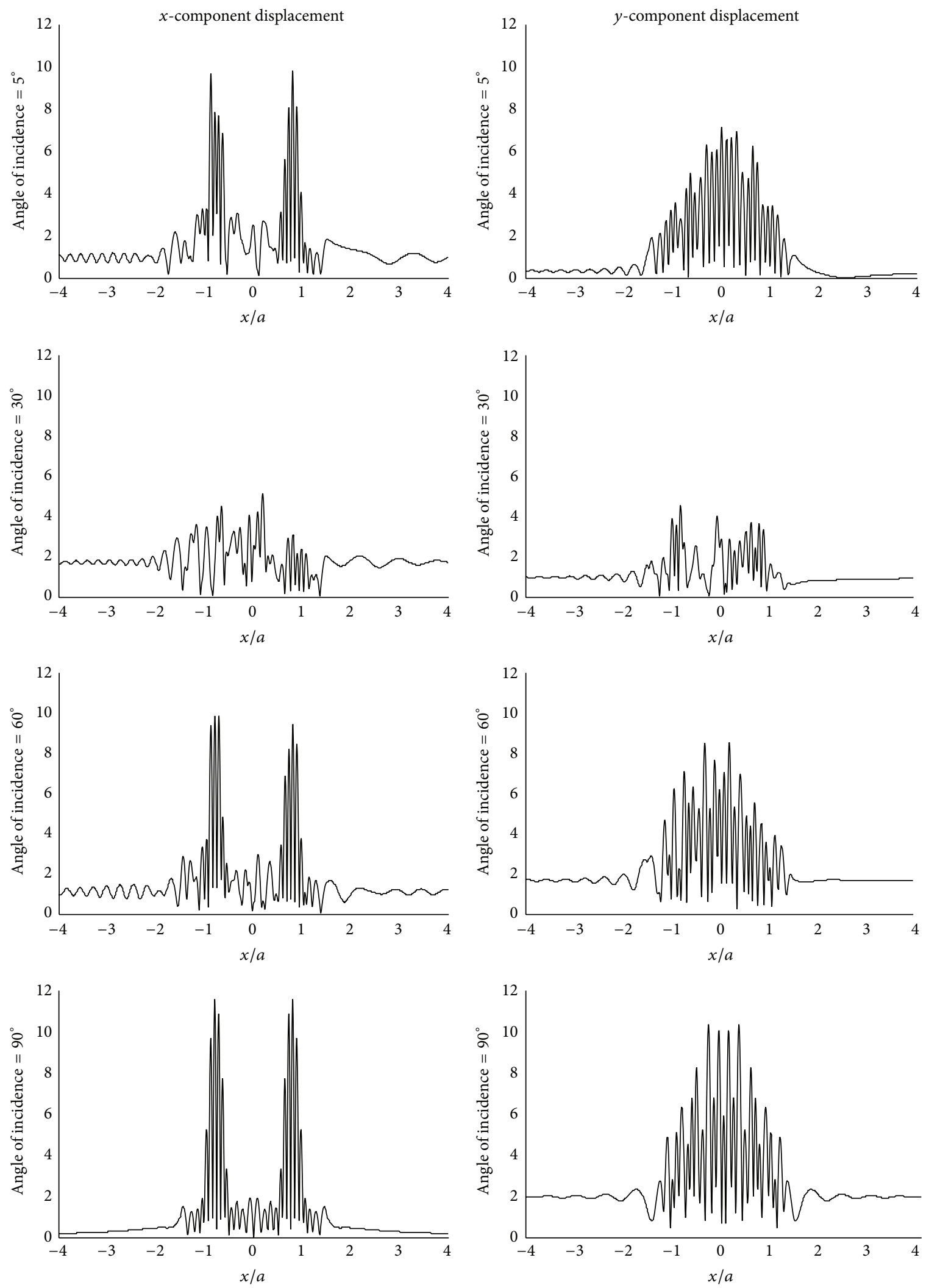

FIGURE 15: Trapezoid-shaped canyon $h / a=1, \eta=6, \theta=5^{\circ}, 30^{\circ}, 60^{\circ}, 90^{\circ}, N_{\max }=28$, and slope $45^{\circ}$. 
amplitudes are highly oscillatory with clustered spikes at the center of the canyon.

\section{Conclusions}

Results of the current research have been presented for various shaped canyons: semicircular, elliptical, and trapezoidal. These results have been presented in Figures 4-15 and the following general conclusions are proposed:

(1) This is the first time that Lee and Liu's redefined cylindrical-wave function has been used to satisfy the zero-stress boundary condition along the half-space for arbitrary-shaped canyons. The application of the weighted residual method for the solution of the wave function allows for a solution to noncircular arbitrary-shaped canyons.

(2) This analytical approach was applied to a semicircular canyon with $\eta=10, N_{\max }=112$, and $\theta=60^{\circ}$. The results were compared to those of Lee and Liu [6] and shown to match exactly. The same amplification of surface displacements, as seen in previous studies, is also demonstrated in these results.

(3) This analytical approach was applied to ellipticalshaped canyons with varying $b / a$ (depth/width) ratios of 1.25 and 1.5. The resulting plots for the displacement amplitudes are for elliptical canyons with a higher frequency range than those for semicircular canyons in previous studies [14].

(4) The elliptical canyon with $b / a=1.25$ produced total displacement amplitudes on both the $x$ - and $y$ components for $\eta=8$, which were highly oscillatory around the free-field surface on the front side of the canyon, and produced a more shadowy behavior along the backside of the canyon. Within the canyon, there were oscillations with spikes at the rims of the canyon at $x / a=-1$ and $x / a=+1$. The oscillations along the surface became more rapid as $\eta$ increased. Increasing the elliptical depth to $b=1.5$ decreased the oscillations within the canyon surface and produced larger spikes with a greater magnitude at the frontside canyon rim at $x / a=-1$.

(5) This analytical approach was then applied to the trapezoidal canyon. The total displacement amplitudes of both the $x$-and $y$-components on the half-space showed a trend of being oscillatory on the front side of the canyon around the free-field amplitudes. On the backside of the canyon, the $x$-component amplitudes were oscillatory about the free field, but the $y$-component amplitudes produced a shadowy behavior around the free field. Both the $x$ - and $y$ components are highly oscillatory about the canyon surface.

(6) At the trapezoidal canyon rims, spikes in the displacement amplitudes were observed. The spikes are consistent with the spikes that were observed for the semicircular and elliptical canyons. These spikes tend to increase in amplitude at higher dimensionless frequencies.

(7) Changing the slope of the trapezoidal canyon walls from $60^{\circ}$ to $45^{\circ}$ demonstrated that the amplitudes of both the $x$-component and $y$-component appeared sensitive to the angle of incidence and the slope of the canyon sides. The amplification of the $x$-component amplitudes and $y$-component amplitudes changed from the canyon sides to the canyon bottom. The degree of the spikes of the canyon rims also changed.

(8) For the trapezoidal canyon, larger values of $\eta$ (greater than 6) caused convergence problems. Further study is necessary.

(9) This method provides good results for arbitraryshaped canyons in which the radius to the canyon surface is approximately equal to $a$. The solutions became numerically unstable as the radius to the canyon boundary became small, because the Bessel functions caused numerical problems.

(10) The method used in this paper, which is an expansion of Lee and Liu's [6] redefined cylindrical-wave function with the application of the weighted residual method, can be extended to other types of incident waves such as SH and SV, as well as surface Rayleigh waves in an elastic homogeneous half-space.

(11) The method used in this paper can be applied to canyons of any shape, but convergence of the solutions may be difficult. This is in particular true when the irregular canyon has nonsmooth sharp corners, and when parts of the canyon are too shallow being close to the origin. The sequel to this paper will involve arbitrary-shaped canyons that are much shallower in depth from the half-space surface.

(12) This method can next be applied to arbitrary-shaped cylindrical valleys, where the valley medium is softer or harder than that of the half-space.

(13) This method can also be extended for problems involving three-dimensional elastic-wave propagations using the same concept and methodology but (different) three-dimensional spherical Hankel functions instead of the two-dimensional cylindrical Hankel functions here.

(14) For underground arbitrary-shaped cavities, the above concept of weighted residues can be applied, but the stress-free boundary conditions at the half-space surface will have to be addressed and taken care of, in addition to that at the cavity's surface.

\section{Summary}

The two-dimensional diffraction of incident $\mathrm{P}$-waves around an arbitrary-shaped canyon in an elastic half-space is presented in this paper. The scattered wave potentials for the resulting $\mathrm{P}$-waves and $\mathrm{S}$-waves are defined by an infinite series of terms with Hankel functions and only sine terms. Using the zero-stress boundary conditions, a solution is made using 
the weighted residual method to create a set of simultaneous equations for the unknown coefficients for a finite number of terms for the series.

The solution is applied to the traditional semicircular canyon to verify the results and show convergence with a finite number of terms. The method is then applied to canyons of other shapes to demonstrate the versatility of the methodology. This paper demonstrates that good results can be achieved in deep elliptical $(b \geq a)$ canyons with frequencies as high as $\eta=8$. However, the results become unstable for the deep elliptical canyons with $\eta>8$ and for the shallow canyons $(b<a)$. Good results were achieved for the trapezoidal canyons of slopes 60 and 45 .

The method in this paper has versatility and may be applied to other incident waves and to canyons of different shapes. A set of papers have been planned and are ongoing on the same subject area. For example, the work on the solution for the diffraction by shallow arbitrary-shaped canyons has just been completed. Other work planned includes diffraction by arbitrary-shaped fluid and valley media.

\section{Conflict of Interests}

The authors declare that there is no conflict of interests regarding the publication of this paper.

\section{Acknowledgment}

The authors would like to thank Professor Trifunac for a critical review and suggestion resulting in the improvement of the content and presentation of the paper.

\section{References}

[1] M. D. Trifuanc, "A note on scattering of plane SH waves by a semi-cylindrical canyon," International Journal of Earthquake Engineering and Structural Dynamics, vol. 1, pp. 267-281, 1973.

[2] H. L. Wong and M. D. Trifunac, "Scattering of plane SH waves by a semi-cylindrical canyon," International Journal of Earthquake Engineering and Structural Dynamics, vol. 3, pp. 159-169, 1974.

[3] H. Cao and V. W. Lee, "Scattering of plane SH waves by circular cylindrical canyons with variable depth-to-width ratio," European Earthquake Engineering, vol. 3, no. 2, pp. 29-37, 1989.

[4] V. W. Lee and M. D. Trifunac, "Trifunac response of tunnels to incident SH waves," Journal of the Engineering Mechanics Division, vol. 105, no. 4, pp. 643-659, 1979.

[5] V. Gičev, M. D. Trifunac, and N. Orbović, “Translation, torsion, and wave excitation of a building during soil-structure interaction excited by an earthquake SH pulse," Soil Dynamics and Earthquake Engineering, vol. 77, pp. 391-401, 2015.

[6] V. W. Lee and W.-Y. Liu, "Two-dimensional scattering and diffraction of $\mathrm{P}$ - and $\mathrm{SV}$-waves around a semi-circular canyon in an elastic half-space: an analytic solution via a stress-free wave function," Soil Dynamics and Earthquake Engineering, vol. 63, pp. 110-119, 2014.

[7] V. W. Lee and H. Cao, "Diffraction of SV waves by circular cylindrical canyons of various depths," Journal of Engineering Mechanics, vol. 115, no. 9, pp. 2035-2056, 1989.
[8] V. W. Lee and X. Y. Wu, "Application of the weighted residual method to diffraction by $2-\mathrm{D}$ canyons of arbitrary shape, I: incident SH waves," Soil Dynamics and Earthquake Engineering, vol. 13, no. 5, pp. 355-364, 1994.

[9] V. W. Lee and X. Y. Wu, "Method of the weighted residual method to diffraction by $2-\mathrm{D}$ canyons of arbitrary shape, II: incident P, SV, \& rayleigh waves," Soil Dynamics and Earthquake Engineering, vol. 13, no. 5, pp. 365-373, 1994.

[10] J. D. Achenbach, Wave Propagation in Elastic Solids, NorthHolland, Elsevier Science, 1973.

[11] M. Abramowitz and I. A. Stegun, Handbook of Mathematical Functions: With Formulas, Graphs and Mathematical Functions, Dover Publications, New York, NY, USA, 1970.

[12] Y. H. Pao and C. C. Mow, Diffraction of Elastic Waves and Dynamic Stress Concentrations, Crane, Russak \& Company, New York, NY, USA, 1973.

[13] R. F. Harrington, "Matrix methods for field problems," Proceedings of the IEEE, vol. 55, no. 2, pp. 136-149, 1967.

[14] William H. Press, S. A. Teukolsky, W. T. Vetterling, and B. P. Flannery, Numerical Recipes in Fortran 77: The Art of Scientific Computing, Cambridge University Press, Cambridge, UK, 1986. 

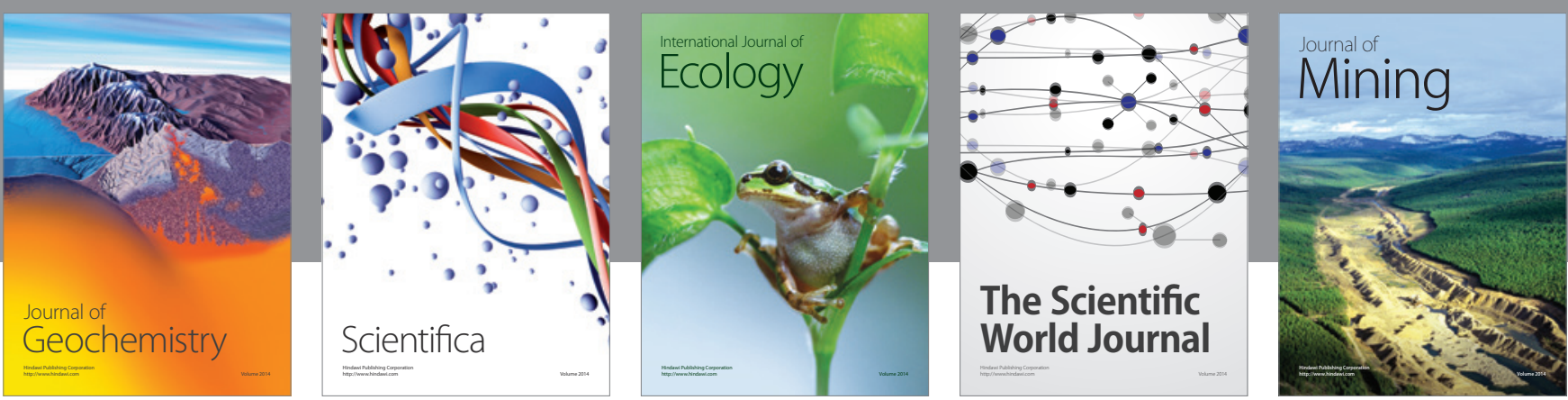

The Scientific World Journal
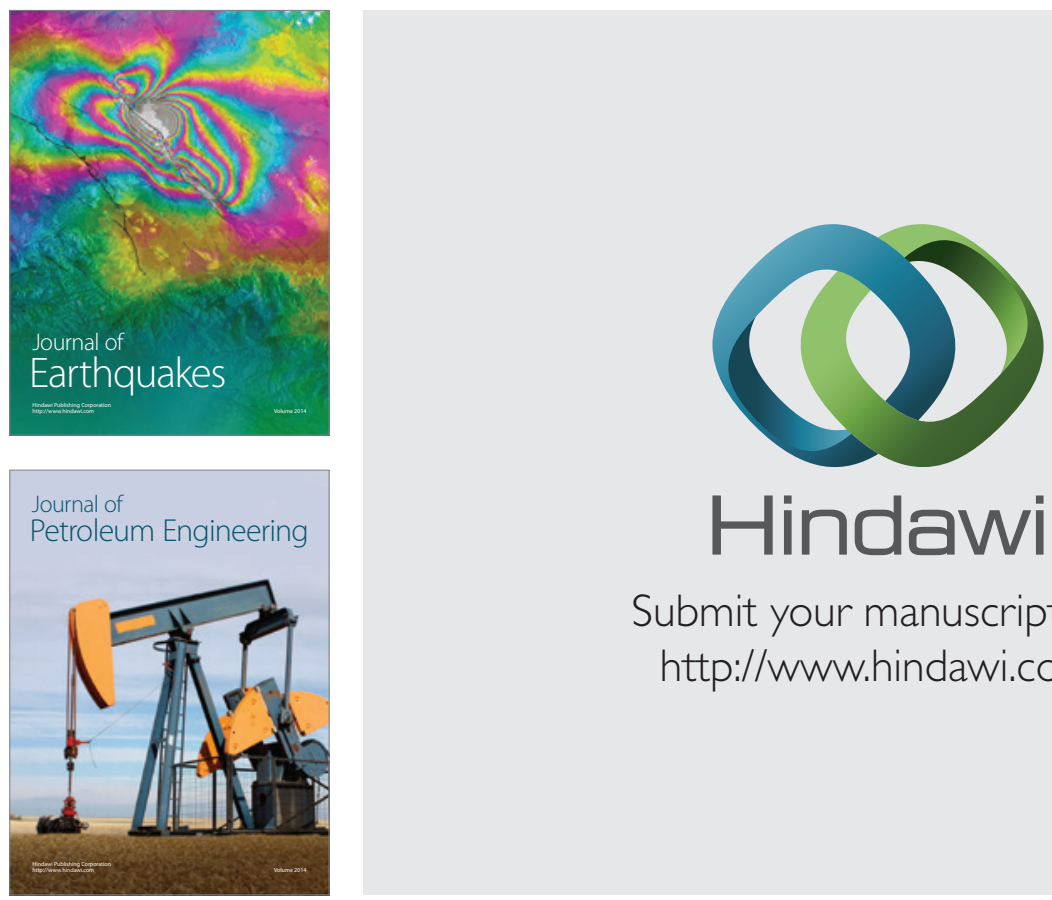

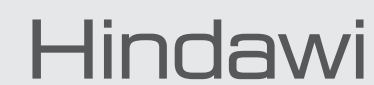

Submit your manuscripts at

http://www.hindawi.com
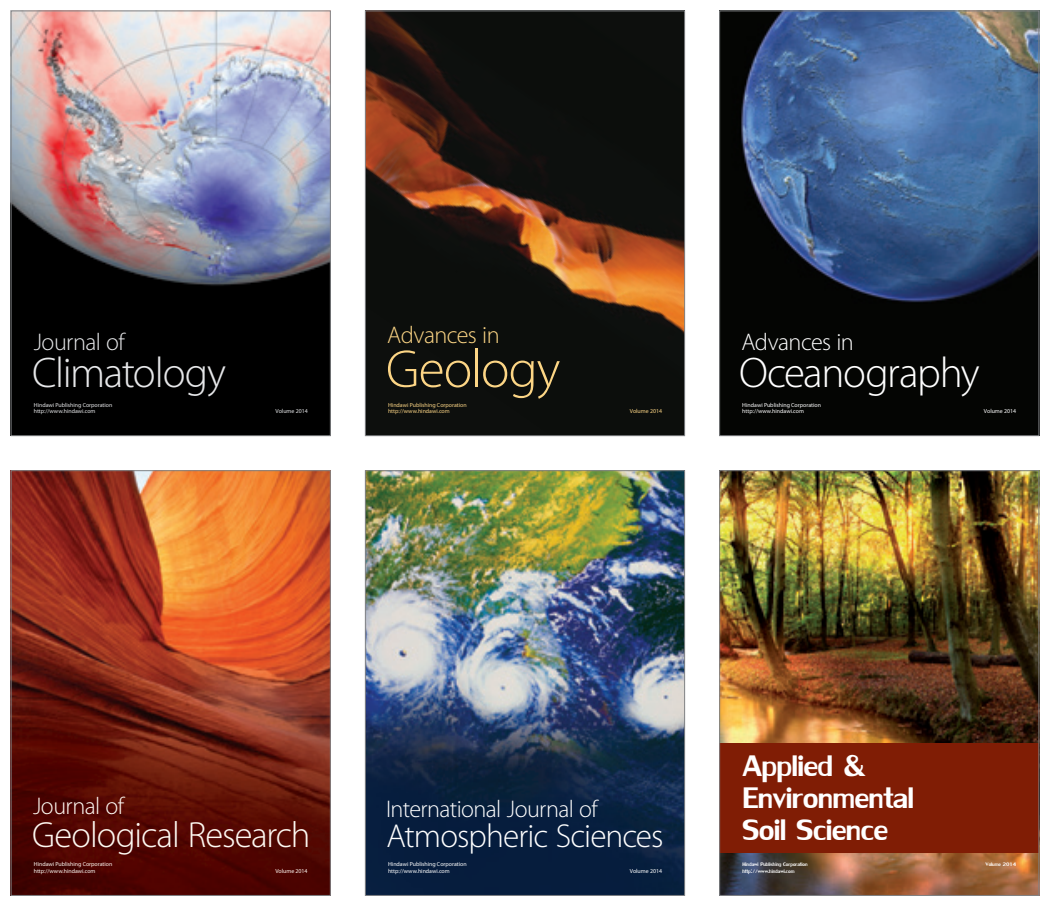
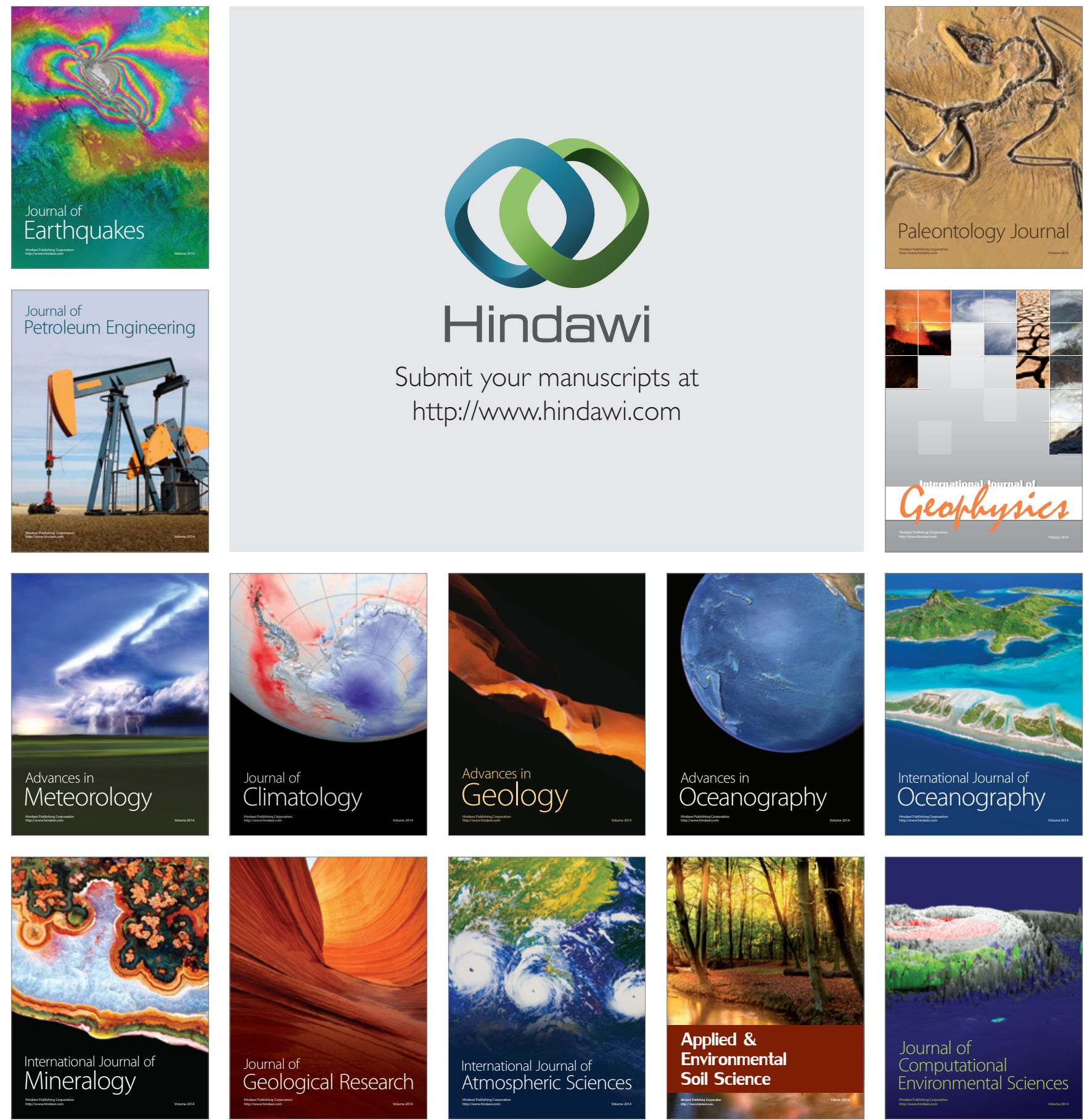